\title{
Biosynthesis of Novel Naphthoquinone Derivatives in the Commonly-used Chassis Cells Saccharomyces cerevisiae and Escherichia coli
}

\author{
W. Wu ${ }^{a, c, e}$, S. Wang ${ }^{b}$, H. Zhang ${ }^{a, c, e}$, W. Guo, H. Lu ${ }^{b}$, H. Xu $u^{a, c, e}$, \\ R. Zhan ${ }^{a, c, e}$, O. Fidan ${ }^{d, *}$, and L. Sun ${ }^{a, c, e, * *}$ \\ ${ }^{a}$ Research Center of Chinese Herbal Resource Science and Engineering, Guangzhou University of Chinese Medicine, \\ Guangzhou, 510006 P. R. China \\ ${ }^{b}$ Suzhou Institute of Drug Control, Suzhou, 215000 P. R. China \\ ${ }^{c}$ Key Laboratory of Chinese Medicinal Resource from Lingnan (Guangzhou University of Chinese Medicine), \\ Ministry of Education, Guangzhou, 510006 P. R. China \\ ${ }^{d}$ Department of Bioengineering, Faculty of Life and Natural Sciences, Abdullah Gül University, Kayseri, 38080 Turkey \\ ${ }^{e}$ Joint Laboratory of National Engineering Research Center for the Pharmaceutics of Traditional Chinese Medicines, \\ Guangzhou, 510006 P. R. China \\ ${ }^{f}$ Artemisinin Research Center, Guangzhou University of Chinese Medicine, Guangzhou, 510405 P. R. China \\ *e-mail: ozkan.fidan@agu.edu.tr \\ **e-mail: sunlei@gzucm.edu.cn
}

Received August 2, 2021; revised August 20, 2021; accepted August 30, 2021

\begin{abstract}
Naphthoquinones harboring 1,4-naphthoquinone pharmacophore are considered as privileged structures in medicinal chemistry. In pharmaceutical industry and fundamental research, polyketide naphthoquinones were widely produced by heterologous expression of polyketide synthases in microbial chassis cells, such as Saccharomyces cerevisiae and Escherichia coli. Nevertheless, these cell factories still remain, to a great degree, black boxes that often exceed engineers' expectations. In this work, the biotransformation of juglone or 1,4-naphthoquinone was conducted to generate novel derivatives and it was revealed that these two naphthoquinones can indeed be modified by the chassis cells. Seventeen derivatives, including 6 novel compounds, were isolated and their structural characterizations indicated the attachment of certain metabolites of chassis cells to naphthoquinones. Some of these biosynthesized derivatives were reported as potent antimicrobial agents with reduced cytotoxic activities. Additionally, molecular docking as simple and quick in silico approach was performed to screen the biosynthesized compounds for their potential antiviral activity. It was found that compound 11 and 17 showed the most promising binding affinities against Nsp9 of SARS-CoV-2, demonstrating their potential antiviral activities. Overall, this work provides a new approach to generate novel molecules in the commonly used chassis cells, which would expand the chemical diversity for the drug development pipeline. It also reveals a novel insight into the potential of the catalytic power of the most widely used chassis cells.
\end{abstract}

Keywords: naphthoquinones, chassis cells, Saccharomyces cerevisiae, Escherichia coli, antiviral, molecular docking, Nsp9 of SARS-CoV-2

DOI: $10.1134 / \mathrm{S} 0003683821100124$

Naphthoquinones, widely reported as 1,4-naphthoquinones, bear 2 carbonyl oxygen atoms and are considered as privileged structures in medicinal chemistry [1]. 1,4-naphthoquinone pharmacophore allows chemical modifications and is suitable scaffold for the synthesis and biosynthesis of new compounds. This will not only generate novel compounds, but also expand and/or enhance the biological activities of naphthoquinones, leading to antitumor, molluscicidal, antileishmanial, antiproliferative, antimalarial, antiviral, antibacterial and antifungal activities [2].
Naphthoquinone derivatives can also help to improve pharmacodynamics and absorption, distribution, metabolism, excretion and toxicity properties [3]. Many naturally isolated and synthetic naphthoquinones, typically lawsone, lapachol, shikonin, juglone, plumbagin and menadione (also known as synthetic vitamin $\mathrm{K}_{3}$ ), have long been elucidated to possess impressive biological and pharmacological activities $[1,3]$. For instance, lawsone, extracted from the henna (Lawsonia inermis) leaves, is the main biologically active molecule with high cytotoxic activities 
(a)

(b)

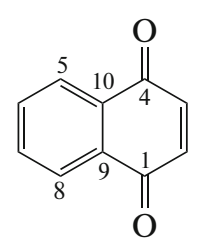

(c)<smiles>[R]NC1=C([R])C(=O)c2c([R3])ccc([R4])c2C1=O</smiles>

Fig. 1. Structures of 1,4-naphthoquinone (a), juglone (b) and naphthoquinone derivatives 1-17 (c).

against disease-causing microorganisms and noted for its antitumor activity [4]. Lapachol, isolated from the bark and wood of Tabebuia sp. and Tecoma sp., was reported as a potential therapeutic agent with various bioactivities such as anti-inflammatory, analgesic, antimalarial, antischistosomal, antiviral, antitrypanosomal, leishmanicide, antifungal, anticancer and antiulcerative activities [5]. Additionally, in a recent study, lapachol and its derivatives were screened against severe acute respiratory syndrome coronavirus 2 (SARS-CoV-2) using an in silico approach and some of lapachol derivatives exhibited strong binding affinity to non-structural protein 9 (Nsp9) of SARS-CoV-2, which is one of the potential drug target proteins [6]. Another natural naphthoquinone is shikonin, which is isolated from Lithospermum erythrorhizon. It was previously announced to be an effective necroptotic inducer for cancer cells [7]. Shikonin was also reported to possess pharmaceutical value in inflammatory and infectious diseases [8]. As a secondary metabolite generated by the walnut tree (Juglandaceae), juglone has several biological activities such as anticancer, antioxidant, antiviral, antibacterial and antifungal effects [9]. Plumbagin, a plant-derived naphthoquinone, is a yellow compound extracted from the root of Plumbago zeylanica $L$. and has a wide range of biological activities including anticancer, anti-fungal, anti-bacterial, anti-malarial, anti-inflammatory, and antimutagenic properties [10].

Vitamin $\mathrm{K}$ is a class of isoprenoid molecules existing in multiple dietary forms, which are essential to normal coagulation as an enzyme cofactor for $\gamma$-carboxylation of peptide-bound Glu residues. All vita$\min \mathrm{K}$ forms are 2-methyl-1,4-naphthoquinone derivatives, term as menaquinones and have important roles in human biology, such as bone, cardiovascular, and metabolic health [11]. As menaquinone is the essential electron-delivery vector of the respiratory chain in various microbial cells, bacteria are the main producers of menaquinone in industry [12]. 1,4-naphthoquinone is the critical intermediate in the biosynthesis of menaquinones in bacteria [13]. Vitamin $\mathrm{K}$ and its derivatives are primarily synthesized by Grampositive bacteria and found in much lower amounts in the food supply in meat, dairy or fermented food products [11].

Engineering microbial systems for the efficient synthesis of natural products has been noted as an important application of synthetic biology. The heterologous reconstitution of the biosynthetic pathways for natural products in microbial chassis cells has attracted increasing attention [14]. Since microbial chassis cells demonstrated high catalytic power of enzymes and a large panoply of biosynthesized products, they have been shown to be profitable in many applications. Some model microorganisms, such as Escherichia coli and Saccharomyces cerevisiae, have been considered as ideal cell factories, thanks to the high growth rate, availability of synthetic biology tools and relatively well-studied metabolic networks [14, 15]. Nevertheless, these cell factories still remain, to a great degree, black boxes that often exceed engineers' expectations. In industrial bioprocesses, different from scientific research, no surprises are desired, and total control over a fully comprehensible and specially designed chassis cell is the ultimate goal. Due to aforementioned bioactivities of naphthoquinones, they are being investigated with respect to their use in therapeutic applications $[1-3]$. This huge potential can be enhanced and expanded by rational modifications to the core structure of naphthoquinones. Besides, compared to the complicated ones, simple naphthoquinones are easy to be modified and optimized by the enzymes of microbial chassis cells [1].

In this work, biotransformation of 1,4-naphthoquinone and juglone in the commonly-used microbial chassis cells, $S$. cerevisiae and E. coli, led to the biosynthesis of a series of "unexpected" naphthoquinone derivatives (Fig. 1 and Table 1). This study not only provided a novel insight into the potential of the catalytic power of these chassis cells, but also expanded the chemical space with the biosynthesis of novel naphthoquinone derivatives. The molecular docking experiments were also conducted to analyze the potential antiviral activities of the biosynthesized compounds and revealed that compound $\mathbf{1 1}$ and $\mathbf{1 7}$ are the most promising antiviral candidates with strong binding affinities to the Nsp9 of SARS-CoV-2.

\section{MATERIALS AND METHODS}

General methods. The metabolites were analyzed and purified on Shimadzu Essentia LC-16 HPLC instrument (Japan) with SPD-16 UV-Vis dual wavelength detector. All UV-spectra were shown in Fig. S1. High-resolution electrospray ionization mass spectroscopy (HRESIMS) measurements were obtained from 1260-6538 Q-TOF system with ZORBAX Eclipse Plus C18 $(2.1 \times 100 \mathrm{~mm} ; 1.8 \mu \mathrm{m})$ column (Agilent Technologies, USA). Linear gradient from 10 to $90 \%$ (vol/vol) acetonitrile-water (containing $0.1 \%$ formic acid) was used for $30 \mathrm{~min}$ at a flow rate of $0.3 \mathrm{~mL} / \mathrm{min}$. NMR spectra were recorded at room temperature on Bruker AVANCE III HD 400 Digital NMR Spectrometer (USA) at $400 \mathrm{MHz}$ for ${ }^{1} \mathrm{H}$ and 
Table 1. The structures of naphthoquinone derivatives (1-17). R1, R2, R3 and R4 in Fig. 1c

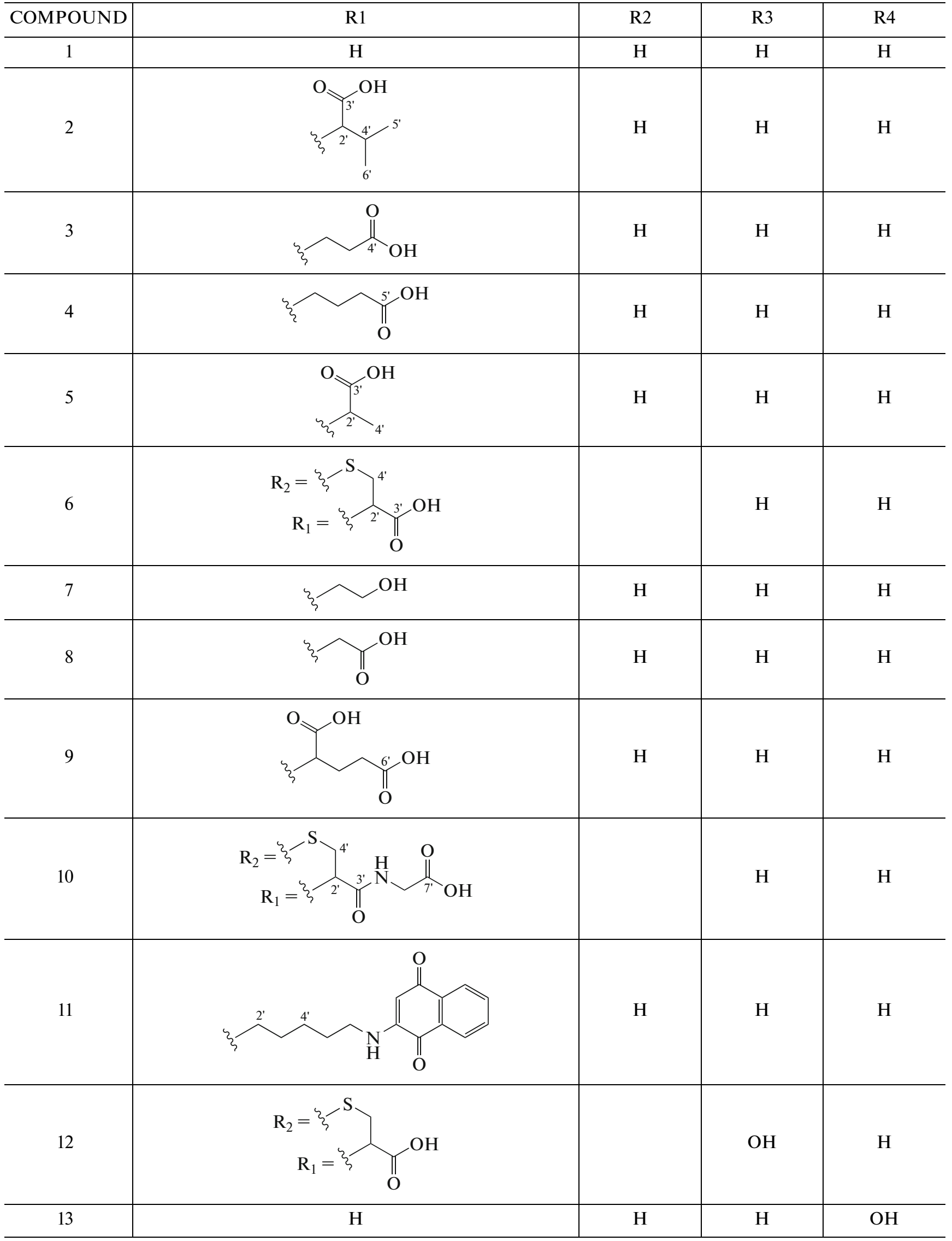


Table 1. (Contd.)

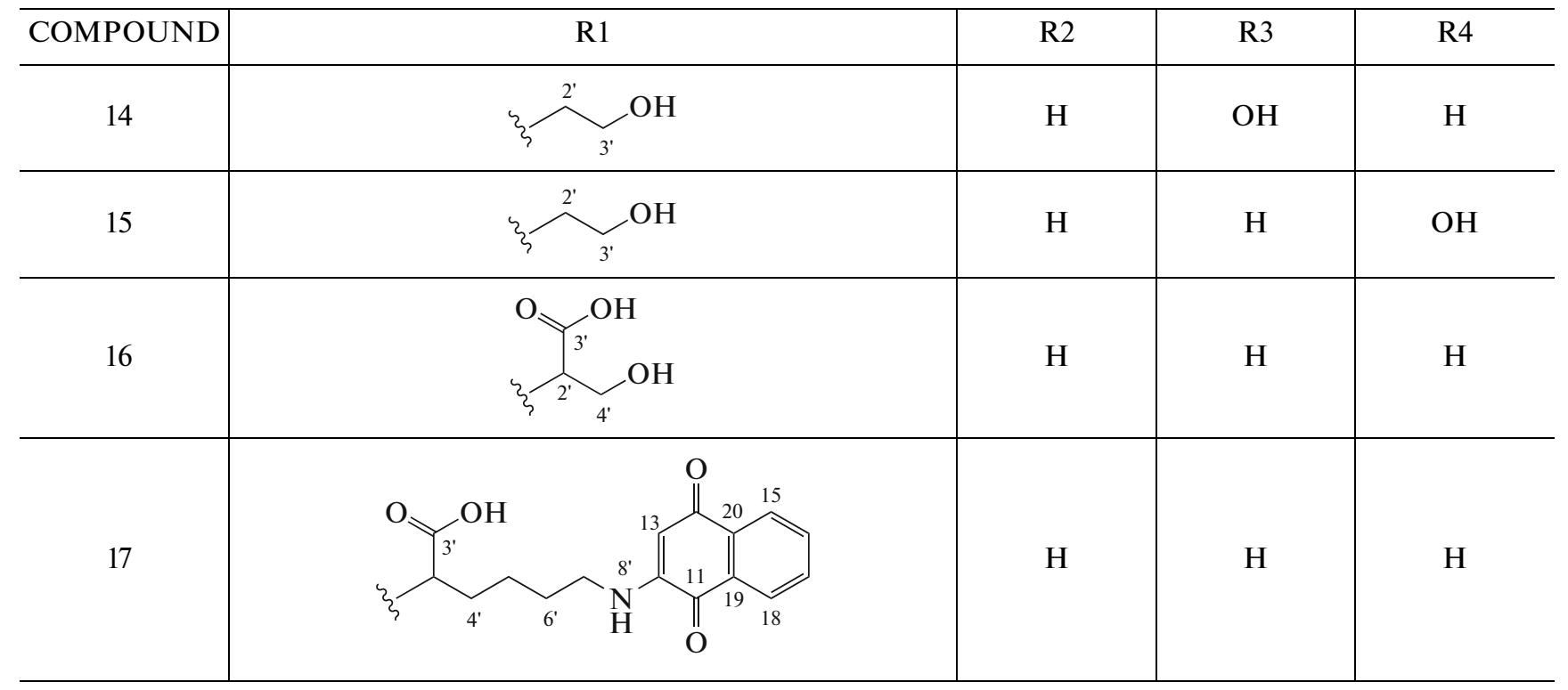

$100 \mathrm{MHz}$ for ${ }^{13} \mathrm{C}$ NMR. The chemical shift $(\delta)$ values are given in parts per million (ppm), and the coupling constants ( $J$ values) are reported in Hertz $(\mathrm{Hz})$. The detailed data of HRESIMS and NMR for each purified compound were listed in Fig. S2-Fig. S55.

Strains and culture conditions. As chassis cells, $S$. cerevisiae INVSc1 and E. coli BL21(DE3) were purchased from Invitrogen (Thermo Fisher Scientific, USA). $S$. cerevisiae INVSc1 was grown in $150 \mathrm{~mL}$ of YPD (1\% yeast extract, 2\% peptone, 2\% dextrose) medium at $30^{\circ} \mathrm{C}$ with shaking at $225 \mathrm{rpm}$ for $24 \mathrm{~h}$. E. coli BL21(DE3) was grown in Luria-Bertani (LB) medium containing $(\mathrm{g} / \mathrm{L})$ : tryptone-10.0, $\mathrm{NaCl}-$ 10.0 , yeast extract $-5.0, \mathrm{pH} 7.0$, at $37^{\circ} \mathrm{C}$ for $24 \mathrm{~h}$ before harvested.

Biosynthesis and characterization of naphthoquinones. 1,4-naphthoquinone (97\%) and juglone (98\%) were purchased from Bide Pharmatech Ltd. (China) and Shanghai Macklin Biochemical Co. Ltd. (China), respectively. L-Glu (99\%), L-Cys (99\%), L-Ser (99\%) and L-Gly (99\%) were obtained from Shanghai Yuanye Bio-Technology Co. Ltd., while L-Lys monohydrochloride (98\%) was purchased from SigmaAldrich (USA). The chassis cells were harvested by centrifugation at $3500 \mathrm{~g}$ for $10 \mathrm{~min}$, washed thrice with $100 \mathrm{mM}$ phosphate buffer (PB, pH 7.4) and transferred to $50 \mathrm{~mL}$ of PB buffer, followed by the supplementation of $20 \mathrm{mg}$ of 1,4-naphthoquinone or $3 \mathrm{mg}$ of juglone. Additionally, $20 \mathrm{mg}$ of corresponding amino acids were added when necessary. To investigate the bioconversion of 6, 16 and 17, chassis cells were resuspended in $1 \mathrm{~mL} \mathrm{~PB}$ buffer, and supplemented with $0.2 \mathrm{mg}$ of substrate. Biotransformation experiment was carried out for 7 days under the same incubation conditions as above. Control groups set as microorganism control (microorganisms in substrate- free PB buffer) and substrate control (substrate in microorganism-free $\mathrm{PB}$ ) were incubated under the same conditions. After fermentation, the broths were extracted thrice with an equal volume of ethyl acetate for $60 \mathrm{~min}$. The extracts were concentrated to dryness under reduced pressure using rotary evaporator (Eyela, Singapore) and the residues were dissolved in methanol. These samples were subsequently analyzed on a Shimadzu Essentia LC-16 instrument (Japan) equipped with column ZORBAX Eclipse Plus C18 $(5 \mu \mathrm{m}, 4.6 \times 250 \mathrm{~mm})$ (Agilent Technologies, USA). Linear gradient from 10 to $90 \%$ (vol/vol) acetonitrilewater (containing $0.1 \%$ formic acid) was used for $30 \mathrm{~min}$ at a flow rate of $1 \mathrm{~mL} / \mathrm{min}$, except for the samples in Fig. 2a, which were analyzed using linear gradient of $20-40 \%$ (vol/vol) for $30 \mathrm{~min}$ and $40-98 \%$ gradient for $10 \mathrm{~min}$ at a flow rate of $0.9 \mathrm{~mL} / \mathrm{min}$.

Purification and structural characterization of 1-17. To isolate the biosynthesized compounds $(\mathbf{1}-\mathbf{1 5})$ the fermentation broths were scaled up to $5 \mathrm{~L}$. After fermentation, the broths were extracted as described above. The metabolites were separated from the extract by a typical purification procedure given below. The crude extract was fractionated on column filled with silica gel 200-300 mesh, eluted with a gradient of hexane-ethyl acetate-methanol $(100: 0: 0,70: 30: 0$, $60: 40: 0,50: 50: 0,40: 60: 0,30: 70: 0,20: 80: 0$, $10: 90: 0,0: 100: 0,0: 50: 50, \mathrm{v} / \mathrm{v}$, each $250 \mathrm{~mL}$ ) to get 10 fractions. The fractions containing the target compounds were further separated by reverse-phase HPLC (ZORBAX Eclipse Plus C18, $5 \mu \mathrm{m}, 4.6 \times 250 \mathrm{~mm}$; (Agilent Technologies, USA), eluted with an isocratic solvent system of acetonitrile-water (containing $0.1 \%$ formic acid) at a flow rate of $1 \mathrm{~mL} / \mathrm{min}$.

To purify compounds 1-6 and 8-10, 1,4-naphthoquinone was incubated with $S$. cerevisiae INVSc1. 
(a)

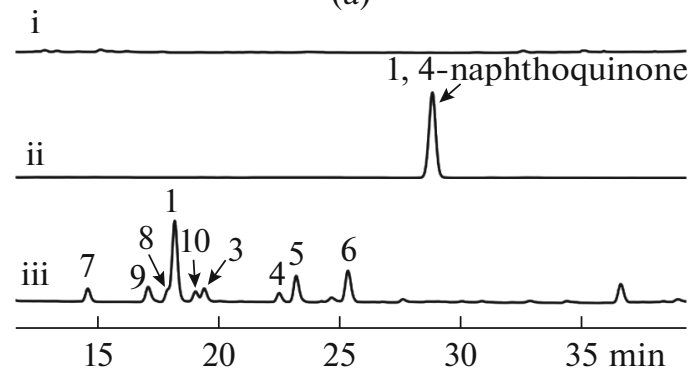

(c)

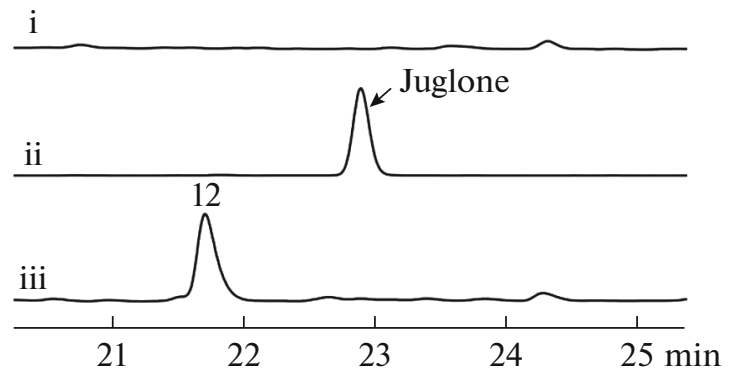

(b)

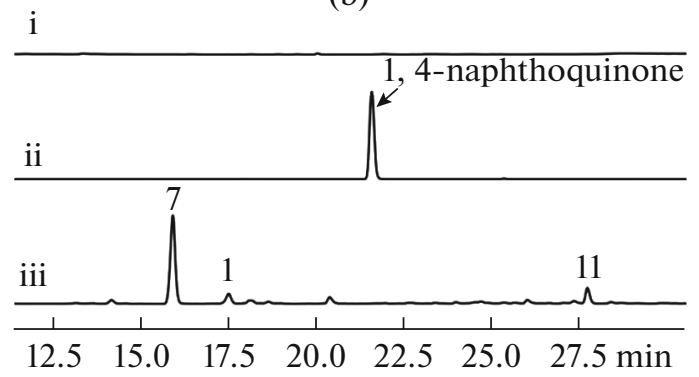

(d)

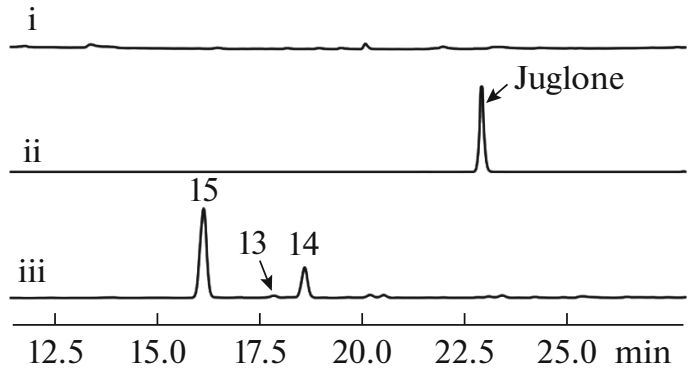

Fig. 2. HPLC (336 nm: (a) and (b); $421 \mathrm{~nm}$ : (c) and (d)) analysis after incubation of the naphthoquinones with chassis cells. (a): i-Culture control of $S$. cerevisiae INVSc1; ii-1,4-naphthoquinone; iii-1,4-naphthoquinone incubated with $S$. cerevisiae INVSc1. (b): i-culture control of E. coli BL21 (DE3); ii-1,4-naphthoquinone; iii-1,4-naphthoquinone incubated with $E$. coli BL21(DE3). (c): i-culture control of $S$. cerevisiae INVSc1; ii-juglone; iii-juglone incubated with $S$. cerevisiae INVSc1. (d): i-culture control of $E$. coli BL21(DE3); ii-juglone; iii-juglone incubated with $E$. coli BL21(DE3). (a) Was analyzed using linear gradient of $20-40 \%$ (vol/vol) acetonitrile-water (containing $0.1 \%$ formic acid) for 30 min and $40-98 \%$ for $10 \mathrm{~min}$ at a flow rate of $0.9 \mathrm{~mL} / \mathrm{min}$, while (b), (c) and (d) were performed at linear gradient of $10-90 \%$ for $30 \mathrm{~min}$ at $1 \mathrm{~mL} / \mathrm{min}$.

After fermentation, the broth was extracted as described above and separated by silica gel column chromatography. The fractions containing the target compounds were further separated by reverse-phase HPLC. The details of purification and structural characterization for the known compounds ( $\mathbf{1}-\mathbf{6}$ and $\mathbf{8}$ ) are provided in the supplementary material.

Compound 9 was found to be in fraction 9 after silica gel column chromatography. It was further purified on HPLC with $17 \%$ acetonitrile-water. The peak at $45 \mathrm{~min}$ was collected to yield $4.7 \mathrm{mg}$ of 9 in pure form. HRESIMS $m / z$ was $304.0819[\mathrm{M}+\mathrm{H}]^{+}$(calculated for $\mathrm{C}_{15} \mathrm{H}_{14} \mathrm{NO}_{6}, 304.0821$ ). Compound 10 was found to be in fraction 10 after silica gel column chromatography. It was further purified on HPLC with $23 \%$ acetonitrile-water. The peak at $16 \mathrm{~min}$ was collected to yield $5.0 \mathrm{mg}$ of $\mathbf{1 0}$ in pure form. HRESIMS $[\mathrm{M}+\mathrm{H}]^{+}$ion signal for $\mathbf{1 0}$ was at $m / z$ 333.0542, which was consistent with the calculated exact mass for $\mathrm{C}_{15} \mathrm{H}_{13} \mathrm{~N}_{2} \mathrm{O}_{5} \mathrm{~S}, 333.0545 .{ }^{1} \mathrm{H},{ }^{13} \mathrm{C}$ and $2 \mathrm{D}$ NMR data are respectively listed in Table 2 and Fig. S22-26.

For the purification of 7 and 11, 1,4-naphthoquinone was incubated with E. coli BL21(DE3). Purification and structural characterization of 7 are provided in the supplementary materials with details. Compound 11 was found to be in fraction 7 after silica gel column chromatography. It was washed with water and methanol after silica gel column chromatography to yield $2.8 \mathrm{mg}$ in pure form. ${ }^{1} \mathrm{H},{ }^{13} \mathrm{C}$ and $2 \mathrm{D}$ NMR data are listed in Table 2 and Fig. S32-36; HRESIMS $[\mathrm{M}+\mathrm{H}]^{+}$ion signal for $\mathbf{1 1}$ was at $m / z$ 415.1659, which perfectly matched with the calculated exact mass for $\mathrm{C}_{25} \mathrm{H}_{23} \mathrm{~N}_{2} \mathrm{O}_{4}, 415.1658$.

To isolate compound $\mathbf{1 2}$, juglone was incubated with $S$. cerevisiae INVSc1. After separation on the silica gel column, 12 was found to be in fraction 10. It was subjected to further purification on HPLC, eluted with $31 \%$ acetonitrile-water. The peak at 20 min was collected, yielding $3.3 \mathrm{mg}$ of $\mathbf{1 2}$ in pure form. ${ }^{1} \mathrm{H},{ }^{13} \mathrm{C}$ and 2D NMR data are given in Table 2 and Fig. S37-41. HRESIMS $[\mathrm{M}+\mathrm{H}]^{+}$ion signal for $\mathbf{1 2}$ was at $m / z$ 292.0276, which was consistent with the calculated exact mass for $\mathrm{C}_{13} \mathrm{H}_{10} \mathrm{NO}_{5} \mathrm{~S}, 292.0280$. To purify 13-15, juglone was incubated with $E$. coli BL21(DE3). The purification and structural characterization of 13-15 are present in the supplementary material.

To isolate 16 and 17, 1,4-naphthoquinone and amino acid were incubated with microorganism. After fermentation, they were extracted as described above. Then the crude extract was fractionated on silica gel column, eluted with a gradient of methanolchloroform $(0: 100,1: 99,2: 98,3: 97,4: 96,5: 95$, $\mathrm{vol} / \mathrm{vol}$, each $250 \mathrm{~mL}$, containing $0.1 \%$ glacial acetic acid) to get 6 fractions. 


\begin{tabular}{|c|c|c|c|c|c|c|c|c|c|c|c|c|c|c|c|c|c|c|c|c|c|c|c|c|c|c|c|c|}
\hline \multirow[t]{2}{*}{$\begin{array}{l}0 \\
0 \\
0 \\
0 \\
\sum^{1} \\
\varrho \\
=\end{array}$} & is & & 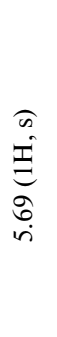 & & 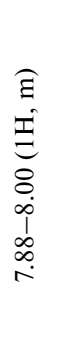 & 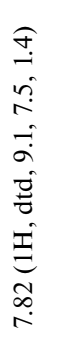 & 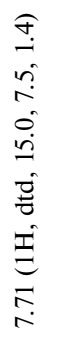 & 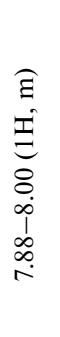 & & & & & 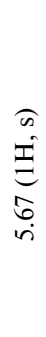 & & $\begin{array}{l}\widehat{\Xi} \\
\mathfrak{I} \\
\Xi \\
8 \\
\dot{0} \\
\dot{\infty} \\
1 \\
\infty \\
\infty\end{array}$ & 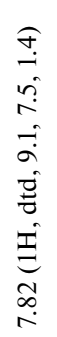 & 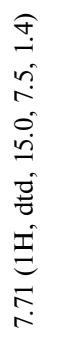 & 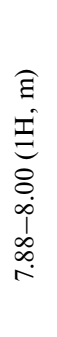 & & & 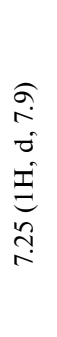 & 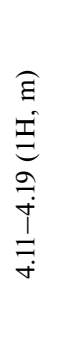 & 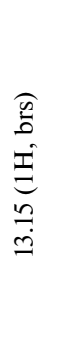 & 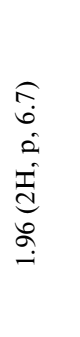 & 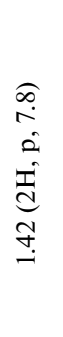 & & 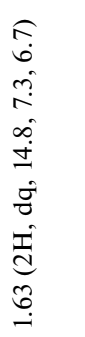 & 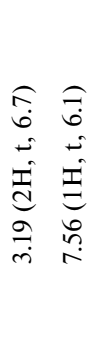 \\
\hline & $\circlearrowright$ & 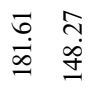 & @̊ & $\frac{\stackrel{\sigma}{\sigma}}{\underline{\infty}}$ & $\begin{array}{l}\cong \\
\stackrel{a}{a}\end{array}$ & $\begin{array}{l}\vec{n} \\
\ddot{n}\end{array}$ & $\begin{array}{l}\infty \\
\stackrel{\infty}{+} \\
\stackrel{\sim}{2}\end{array}$ & 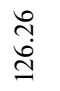 & $\begin{array}{l}0 \\
\stackrel{0}{0} \\
0 \\
0\end{array}$ & $\begin{array}{l}\stackrel{a}{m} \\
\stackrel{m}{a}\end{array}$ & $\underset{\infty}{\stackrel{\infty}{a}}$ & $\begin{array}{l}\infty \\
\infty \\
\infty \\
\dot{J}\end{array}$ & $\frac{\pi}{2}$ & $\begin{array}{l}0 \\
\stackrel{0}{1} \\
\infty\end{array}$ & $\begin{array}{l}\vec{\infty} \\
\varrho\end{array}$ & $\begin{array}{l}\infty \\
n \\
n \\
n\end{array}$ & $\begin{array}{l}2 \\
\infty \\
i \\
n\end{array}$ & $\begin{array}{l}\stackrel{q}{+} \\
\stackrel{0}{0}\end{array}$ & $\begin{array}{l}\stackrel{2}{0} \\
\stackrel{0}{0}\end{array}$ & $\begin{array}{l}\vec{b} \\
\dot{m}\end{array}$ & & $\begin{array}{l}m \\
i n \\
i n\end{array}$ & $\begin{array}{l}\dot{J} \\
\stackrel{\Xi}{\Xi}\end{array}$ & $\begin{array}{l}\stackrel{p}{0} \\
\dot{m}\end{array}$ & $\begin{array}{l}\vec{m} \\
\vec{\lambda}\end{array}$ & & 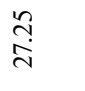 & 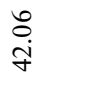 \\
\hline \multirow[t]{2}{*}{$\begin{array}{l}\text { ర0 } \\
1 \\
0 \\
\sum_{0}^{n} \\
0 \\
0 \\
0\end{array}$} & $\omega^{T}$ & & 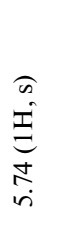 & & 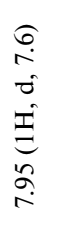 & 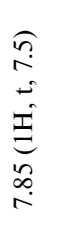 & 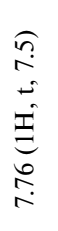 & 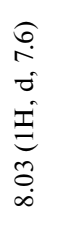 & & & & & & & & & & & & & 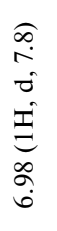 & 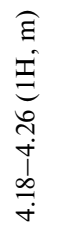 & & 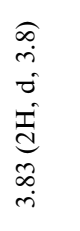 & & & & \\
\hline & $\infty$ & 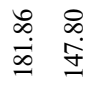 & 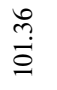 & $\begin{array}{l}\infty \\
\underset{\infty}{\infty} \\
\infty\end{array}$ & $\begin{array}{l}\stackrel{2}{a} \\
\cong\end{array}$ & $\begin{array}{l}\vec{n} \\
\ddot{n}\end{array}$ & $\begin{array}{l}\tilde{a} \\
\tilde{n}\end{array}$ & $\begin{array}{l}\infty \\
\stackrel{\infty}{0} \\
\stackrel{0}{1}\end{array}$ & $\begin{array}{l}n \\
0 \\
0 \\
0 \\
0\end{array}$ & $\begin{array}{l}\dot{m} \\
\dot{m} \\
\ddot{n}\end{array}$ & & & & & & & & & & & & $\stackrel{n}{i}$ & $\stackrel{\text { 巳 }}{\stackrel{\Xi}{E}}$ & @ & & & & \\
\hline \multirow[t]{2}{*}{ 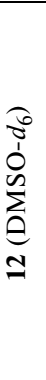 } & $\omega^{T}$ & & & & $\begin{array}{l}\widehat{a} \\
\vec{\Xi} \\
\Xi \\
\vec{\Xi} \\
\dot{\Xi}\end{array}$ & 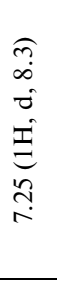 & 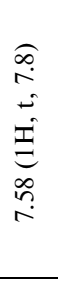 & 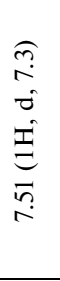 & & & & & & & & & & & & & 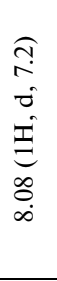 & 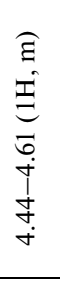 & & 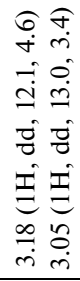 & & & & \\
\hline & $\infty$ & 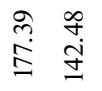 & $\begin{array}{l}\stackrel{8}{0} \\
\dot{0} \\
\stackrel{0}{0}\end{array}$ & $\begin{array}{l}\stackrel{\text { I }}{+} \\
\stackrel{\infty}{\infty}\end{array}$ & $\begin{array}{l}\overrightarrow{0} \\
\dot{0} \\
0\end{array}$ & $\begin{array}{l}n \\
\ddot{n} \\
\cong\end{array}$ & $\begin{array}{l}\stackrel{0}{N} \\
\stackrel{n}{n}\end{array}$ & $\begin{array}{l}\ddot{n} \\
\stackrel{\Xi}{=}\end{array}$ & $\begin{array}{l}\stackrel{\Im}{+} \\
\stackrel{0}{0}\end{array}$ & $\begin{array}{l}\vec{J} \\
\stackrel{\Xi}{\Xi}\end{array}$ & & & & & & & & & & & & $\begin{array}{l}\dot{+} \\
\stackrel{n}{n}\end{array}$ & $\stackrel{ }{\stackrel{5}{\Xi}}$ & ले & & & & \\
\hline \multirow[t]{2}{*}{$\begin{array}{l}0 \\
i \\
D \\
0 \\
0 \\
0 \\
0 \\
0 \\
= \\
=\end{array}$} & is & & $\begin{array}{l}\widehat{a} \\
\vec{\Xi} \\
\Xi \\
\stackrel{m}{\Xi} \\
\stackrel{n}{n}\end{array}$ & & 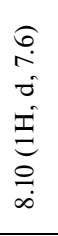 & 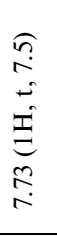 & 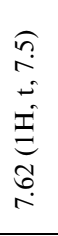 & 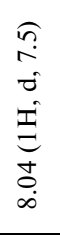 & & & & & & & & & & & & & $\begin{array}{l}\widehat{\Xi} \\
\tilde{I} \\
\Xi \\
m \\
\vdots \\
i \\
1 \\
0 \\
0 \\
i\end{array}$ & 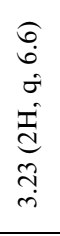 & 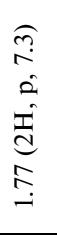 & 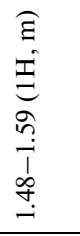 & & & & \\
\hline & $\infty$ & 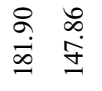 & $\begin{array}{l}\hat{\alpha} \\
\dot{8}\end{array}$ & $\begin{array}{l}\text { ळે } \\
\infty\end{array}$ & 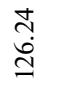 & $\begin{array}{l}\stackrel{ }{\infty} \\
\dot{+} \\
\end{array}$ & $\begin{array}{l}\tilde{o} \\
\stackrel{i}{2}\end{array}$ & $\begin{array}{l}m \\
\\
\stackrel{0}{1}\end{array}$ & $\begin{array}{l}\stackrel{0}{n} \\
\stackrel{n}{n} \\
n\end{array}$ & $\begin{array}{l}n \\
\dot{b} \\
\dot{n} \\
n\end{array}$ & & & & & & & & & & & & $\stackrel{n}{n}$ & $\begin{array}{l}\tilde{o} \\
\infty \\
\infty \\
\sim\end{array}$ & $\begin{array}{l}30 \\
\dot{d}\end{array}$ & & & & \\
\hline \multirow[t]{2}{*}{$\begin{array}{l}\text { To } \\
1 \\
0 \\
0 \\
\sum_{1}^{0} \\
0 \\
0\end{array}$} & it & & & & 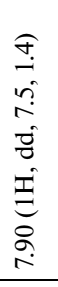 & 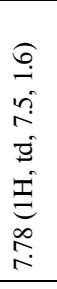 & 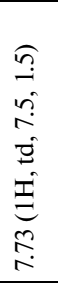 & 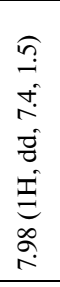 & & & & & & & & & & & & & 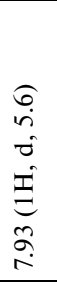 & 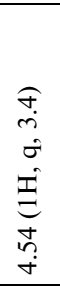 & & 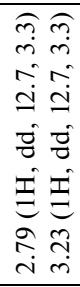 & & 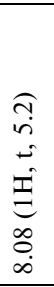 & 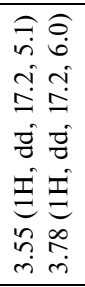 & \\
\hline & $\infty$ & $\begin{array}{ll}\stackrel{\infty}{\infty} & \infty \\
\stackrel{+}{=} & \stackrel{+}{ \pm} \\
\end{array}$ & $\begin{array}{l}\tilde{N} \\
\stackrel{\Xi}{\Xi}\end{array}$ & $\begin{array}{l}\text { ర్ } \\
\stackrel{\infty}{=} \\
=\end{array}$ & $\begin{array}{l}\infty \\
\varrho \\
\beth\end{array}$ & $\begin{array}{l}\tilde{D} \\
\dot{D} \\
\stackrel{+}{ }\end{array}$ & $\begin{array}{l}\stackrel{a}{\vec{m}} \\
\stackrel{-}{=}\end{array}$ & 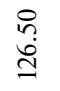 & $\begin{array}{l}\stackrel{N}{0} \\
\stackrel{0}{=}\end{array}$ & 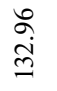 & & & & & & & & & & & & $\vec{m}$ & $\begin{array}{l}\text { bे } \\
\text { فे }\end{array}$ & 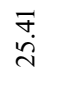 & & & $\begin{array}{l}\stackrel{J}{~} \\
\text { i }\end{array}$ & $\stackrel{F}{\vec{\Xi}}$ \\
\hline \multirow[t]{2}{*}{ 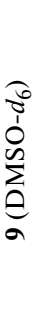 } & is & & 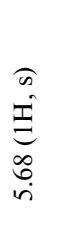 & & 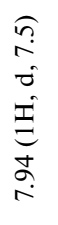 & 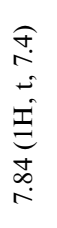 & 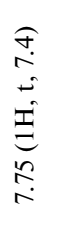 & 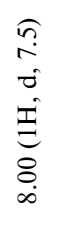 & & & & & & & & & & & & & 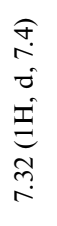 & 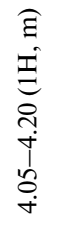 & 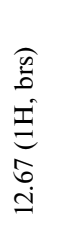 & 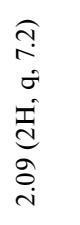 & 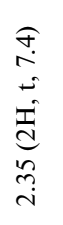 & & 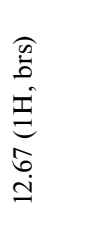 & \\
\hline & $\infty$ & 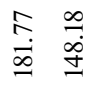 & $\begin{array}{l}\stackrel{\circ}{\dot{0}} \\
\text { o }\end{array}$ & $\begin{array}{l}\infty \\
\stackrel{\infty}{\sigma} \\
\infty\end{array}$ & 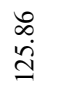 & $\begin{array}{l}\stackrel{q}{+} \\
\stackrel{n}{n}\end{array}$ & $\begin{array}{l}\hat{\infty} \\
\stackrel{i}{a}\end{array}$ & $\begin{array}{l}\infty \\
\stackrel{+}{0} \\
\stackrel{\Xi}{\Xi}\end{array}$ & $\begin{array}{l}\stackrel{2}{0} \\
\dot{0} \\
0\end{array}$ & $\begin{array}{l}\hat{m} \\
\ddot{n}\end{array}$ & & & & & & & & & & & & $\stackrel{n}{\stackrel{n}{+}}$ & $\begin{array}{l}\vec{n} \\
\stackrel{i}{\Xi}\end{array}$ & సి & 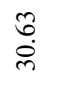 & & $\begin{array}{l}\stackrel{+}{+} \\
\stackrel{+}{I}\end{array}$ & \\
\hline 2 & & $-\quad r$ & $m$ & $\nabla$ & $n$ & 0 & $r$ & $\infty$ & $a$ & $ㅇ$ & $=$ & $\simeq$ & & \pm & $\bumpeq$ & $\underline{0}$ & $=$ & $\cong$ & 9 & 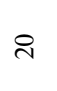 & $\begin{array}{l}I_{Z} \\
Z_{-1}^{\prime}\end{array}$ & $i$ & $m$ & i & in & & 6 & $\therefore \underset{-\infty}{\mathbf{Z}}$ \\
\hline
\end{tabular}


To isolate 16, 1,4-naphthoquinone and L-Ser were incubated with $S$. cerevisiae INVSc1. The broth was scaled up to $300 \mathrm{~mL}$. After separation on the silica gel column, 16 was found to be in fraction 6, which was further separated by HPLC with $20 \%$ acetonitrilewater (containing $0.1 \%$ formic acid) at a flow rate of $1 \mathrm{~mL} / \mathrm{min}$. The peak at $11.13 \mathrm{~min}$ was collected to yield $1.6 \mathrm{mg}$ of 16 in pure form. ${ }^{1} \mathrm{H},{ }^{13} \mathrm{C}$ and $2 \mathrm{D}$ NMR data are found in Table 2 and Fig. S46-50; HRESIMS $[\mathrm{M}+\mathrm{H}]^{+}$ion signal was found at $m / z$ 262.0711, which matched with the calculated exact mass for $\mathrm{C}_{13} \mathrm{H}_{12} \mathrm{NO}_{5}$, 262.0715.

To isolate 17, 1,4-naphthoquinone and L-Lys monohydrochloride were incubated with $E$. coli BL21(DE3). The broth was scaled up to $2 \mathrm{~L}$. After separation on the silica gel column, 17 was found to be in fraction 6, which was washed with water and methanol after dryness under vacuum. Twenty $\mathrm{mg}$ of $\mathbf{1 7}$ in pure form were obtained. ${ }^{1} \mathrm{H},{ }^{13} \mathrm{C}$ and $2 \mathrm{D}$ NMR data are found in Table 2 and Fig. S51-55; HRESIMS $[\mathrm{M}+\mathrm{H}]^{+}$ion signal was at $m / z$ 459.1561, which was consistent with calculated exact mass for $\mathrm{C}_{26} \mathrm{H}_{23} \mathrm{~N}_{2} \mathrm{O}_{6}, 459.1556$.

Molecular Docking. The crystal structure of Nsp9 (PDB ID: 6W4B) was downloaded from the protein data bank (PDB) of Research Collaboratory for Structural Bioinformatics (RCSB). The crystal structure of the protein was determined at $2.95 \AA$ resolution [16]. The ligands are first drawn in ChemDraw Professional 16.0, followed by MM2 minimization using Chem3D and saved as SDF files. Subsequently, the SDF files were converted PDB files for each ligand using Open Babel GUI software. Both protein and the ligands were prepared for the molecular docking experiments using AutoDock Tools (ADT) 1.5.6 [17]. In the protein preparation process, water molecules were deleted, polar hydrogen atoms and Kollmans charges were added. For ligands, Gasteiger charges were added, aromatic carbon and rotatable bonds were detected, torsion number was automatically set, and non-polar hydrogens were merged. Then, protein and ligand files were saved as PDBQT files. Druggable pockets of the protein was predicted using online CavityPlus platform (http://www.pkumdl.cn:8000/cavityplus/computation.php). Based on the predicted most druggable pockets, the grid boxes were determined using ADT software. The grid box for pocket 1 was centered at $x=39.82, y=-19.72$ and $z=24.30$ with sizes of 22.28, 22.17 and $24.69 \AA$. For the pocket 2, grid box was centered at $x=56.88, y=-0.77$ and $z=23.96$ with the sizes of $25.97,26.32$ and $26.86 \AA$. Finally, the prepared flexible ligands were docked against the fixed rigid 6W4B protein using Auto Vina in Windows command line window after preparing config files with necessary information (receptor, grid box coordinates, number of modes: 10, energy range: 8 and exhaustiveness: 16) [18]. Pymol version 2.4.1 and Discovery Studio 2020 Client software were used for the visualization and protein-ligand interactions, respectively.

\section{RESULTS AND DISCUSSION}

Products analysis of the naphthoquinones incubated with chassis cells. Our previous research has revealed that supplement of different nutrients into the fermentation broth of engineered E. coli resulted into the biosynthesis of unexpected by-products [19], suggesting the enzymes of host microorganisms may modify the structures of final products. To investigate the potential catalytic power of chassis cells, 2 naphthoquinones, 1,4-naphthoquinone and juglone, were added, respectively, into the $\mathrm{PB}$ buffer containing the most widely used chassis cells, $S$. cerevisiae or E. coli. After 7 days of incubation, the broths were extracted and subjected to HPLC analysis. In the broth containing $S$. cerevisiae INVSc1 and 1,4-naphthoquinone as substrate, the extract revealed the presence of serial new product peaks 1-10 compared to empty control (Fig. 2a). 1,4-naphthoquinone incubated with $E$. coli BL21(DE3) was bioconverted to compounds 1, 7 and 11 (Fig. 2b). Although juglone, noted as 5-hydroxy1,4-naphthoquinone, shared a very similar structure with 1,4-naphthoquinone, $\mathbf{1 2}$ was the only major product of the biotransformation of $S$. cerevisiae INVSc1 (Fig. 2c). When juglone incubated with E. coli BL21(DE3), compounds 13-15 were detected (Fig. 2d). These results suggested that a series of naphthoquinone derivatives were generated when the naphthoquinones incubated with chassis cells.

Structural characterization of compounds 1-15. Compounds $\mathbf{1}-\mathbf{1 5}$ possess similar UV-vis spectra, suggesting that they have similar structures (Fig. S1). For the identification of the chemical structures of 1-15, $S$. cerevisiae INVSc1 or E. coli BL21(DE3) cells were harvested in large scale supplemented with 1,4-naphthoquinone or juglone. The compounds were purified by open column chromatography and HPLC. For the known compounds, structure characterization was conducted by the comparison of the published HRESIMS data and NMR spectra with the ones obtained in this work, which are provided in the supplementary material.

Compound 9 was obtained as a red powder. The molecular formula of 9 was determined as $\mathrm{C}_{15} \mathrm{H}_{13} \mathrm{NO}_{6}$ based on the HRESIMS protonated molecular ion at $m / z$ 304.0819. Comparison of ${ }^{1} \mathrm{H}$ NMR data of 9 with that of the substrate 1,4-naphthoquinone (Fig. S3 and S22) revealed 4 aromatic proton signals $(\delta 8.00$, $7.94,7.84$, and 7.75$)$ and a $\mathrm{CH}$ singlet $(\delta 5.68)$ indicating the presence of naphthoquinone moiety and the substitution of one proton for quinone ring. The additional proton signals, including an overlapped signal corresponding to 2 carboxyl protons $(\delta 12.67), \mathrm{NH}$ doublet belonging to $\mathrm{NH}-1^{\prime}(\delta 7.32), \mathrm{CH}$ multiplet belonging to $\mathrm{H}-2^{\prime}(\delta 4.05-4.20), \mathrm{CH} 2$ triplet belonging to $\mathrm{H}^{-5}{ }^{\prime}(\delta 2.35)$ and $\mathrm{CH} 2$ quartet belonging to $\mathrm{H}-4^{\prime}$ $(\delta$ 2.09), established the presence of Glu residue based on the ${ }^{1} \mathrm{H}-{ }^{1} \mathrm{H}$ COSY correlations among $\mathrm{NH}-1$ ', $\mathrm{H}-2$ ', $\mathrm{H}-4^{\prime}$ and $\mathrm{H}-5^{\prime}$ (Fig. 3 and S24). Consistently, the 

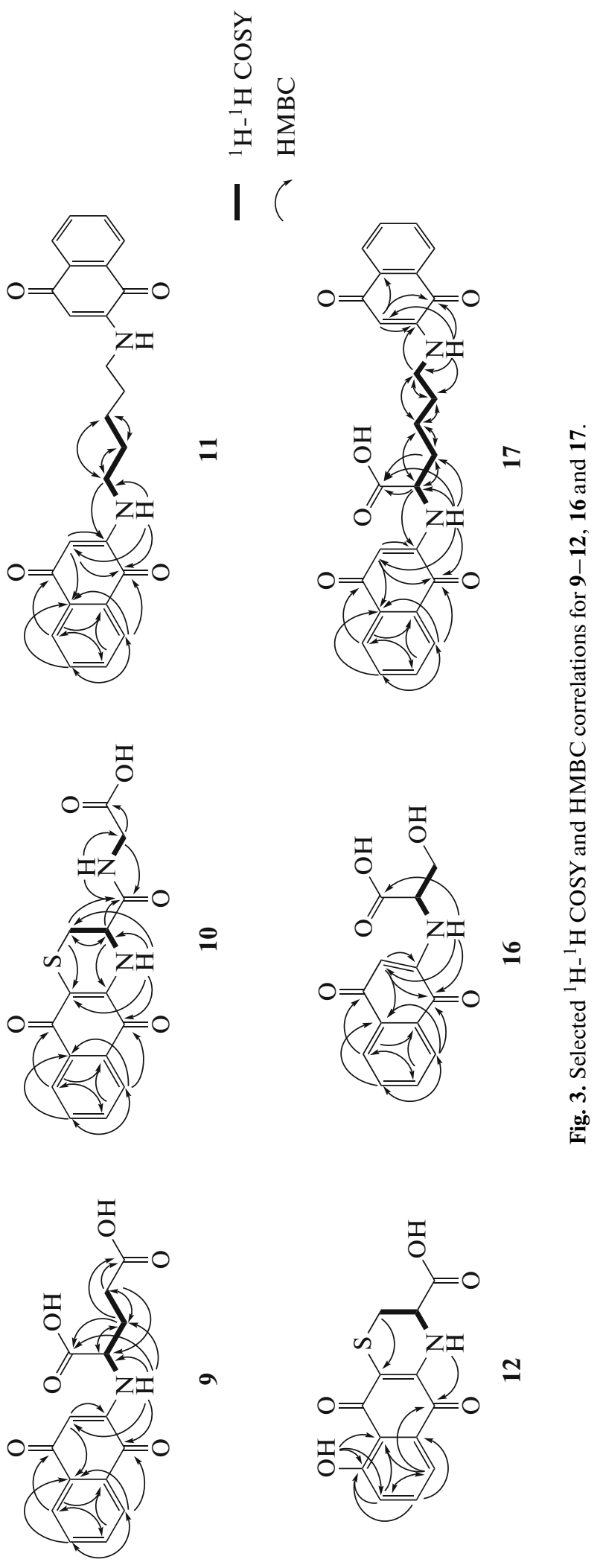

${ }^{13} \mathrm{C}$ NMR spectrum of 9 exhibited signals attributable to a naphthoquinone, 2 carboxyl groups $(\delta 174.54$ and $172.51)$, a methine group $(\delta 54.73)$ and 2 methylene groups $(\delta 30.63$ and 26.26) (Fig. S23). Although the carbon signal at $\delta 172.51$ was weak, we identified it through the heteronuclear multiple-bond correlation (HMBC) spectrum (Fig. S26). These findings suggested that Glu group was introduced to the quinone ring of 1,4-naphthoquinone at $\mathrm{C}-2$. This was confirmed by the HMBC spectrum. As shown in Fig. 3, the HMBC correlations of $\mathrm{NH}-1^{\prime}$ at $\delta 7.32$ to $\mathrm{C}-1, \mathrm{C}-3$, C-2', C-3' and C-4', H-2' at $\delta$ 4.05-4.20 to C-4', H-4' at $\delta 2.09$ to $\mathrm{C}-2^{\prime}, \mathrm{C}-3^{\prime}, \mathrm{C}-5^{\prime}$ and $\mathrm{C}-6^{\prime}$, and $\mathrm{H}-5^{\prime}$ at $\delta 2.35$ to C-2', C-4' and C-6' confirmed the connection of the Glu group with 1,4-naphthoquinone at C-2. Thus, compound 9 was identified as (1,4-dioxo-1,4dihydronaphthalen-2-yl)glutamic acid. The assigned carbon and proton signals are listed in Table 2.

Compound 10 was isolated as a purple powder with determined the molecular formula as $\mathrm{C}_{15} \mathrm{H}_{12} \mathrm{~N}_{2} \mathrm{O}_{5} \mathrm{~S}$ according to its HRESIMS data $[\mathrm{M}+\mathrm{H}]^{+}, m / z 333.0542$. This was 57 mass units larger than compound $\mathbf{6}$, which possessed identical UV-vis spectra with it (Fig. S1). Similar to that of 6 , the ${ }^{1} \mathrm{H}$ NMR and ${ }^{1} \mathrm{H}-{ }^{1} \mathrm{H}$ COSY spectra of 10 (Fig. S13, S15, S27 and S29) also showed the presence of a naphthoquinone core $(\delta 7.98,7.90$, 7.78, and 7.73) and Cys moiety bridging $\mathrm{C}-2$ and $\mathrm{C}-3$ of the quinone ring $(\delta 7.93,4.54,2.79$, and 3.23$)$, excepting 3 additional proton signals, including NH triplet at $\delta 8.08$ and 2 vicinal $\mathrm{CH}$ doublet of doublets at $\delta 3.55$ and $\delta 3.78$, respectively. Besides, 2 additional carbon signals were found in the ${ }^{13} \mathrm{C}$ NMR spectrum, $\delta 42.04$ and 171.41 (Fig. S14 and S28). According to the heteronuclear single quantum coherence (HSQC) spectrum, both of the two $\mathrm{CH}$ protons $(\delta 3.55$ and 3.78) were directly attached to the carbon at $\delta 42.04$ (Fig. S30). All of these data suggested that $\mathbf{1 0}$ was a derivative of $\mathbf{6}$ and may contain an additional Gly residue connected to the Cys via an amide bond. It was confirmed by the HMBC correlations shown in Fig. 3. The HMBC correlations of $\mathrm{NH}-1$ ' at $\delta 7.93$ to $\mathrm{C}-1, \mathrm{C}-3$, C-2' and C-4', H-2' at $\delta 4.54$ to C-2, C-3' and C-4', and $\mathrm{H}-4^{\prime}$ at $\delta 2.79$ and 3.23 to $\mathrm{C}-3, \mathrm{C}-2^{\prime}$ and $\mathrm{C}-3^{\prime}$ confirmed the connection of the Cys residue group with 1,4-naphthoquinone at C-2 and C-3. The linkage of Gly residue to Cys group and the peptide bond were determined based on the HMBC correlations of NH-5' at $\delta 8.08$ to C-3' and C-6', and H-6' at $\delta 3.55$ and 3.78 to $\mathrm{C}-3^{\prime}$ and $\mathrm{C}-\mathbf{7}^{\prime}$. Therefore, $\mathbf{1 0}$ was identified as (5,10-dioxo-3,4,5,10-tetrahydro-2H-naphtho[2,3-b] $[1,4]$ thiazine-3-carbonyl)glycine. All the carbon and proton signals were assigned and are listed in Table 2 .

Compound 11 was isolated as an orange powder, exhibiting the molecular formula $\mathrm{C}_{25} \mathrm{H}_{22} \mathrm{~N}_{2} \mathrm{O}_{4}$ as established by HRESIMS ([M $+\mathrm{H}]^{+}, m / z$ 415.1659). It was clearly a symmetric structure as judged by its ${ }^{1} \mathrm{H}$ and ${ }^{13} \mathrm{C}$ NMR spectra (Fig. S32 and S33), which showed 11 protons and 13 carbons. In the ${ }^{1} \mathrm{H}$ NMR 
spectrum, 4 aromatic proton signals ( $\delta 8.10,8.04$, 7.73 , and 7.62), a NH multiplet $(\delta 5.86-5.93)$ and a $\mathrm{CH}$ singlet $(\delta 5.73)$ observed in the low field showed the presence of a naphthoquinone core and the substitution of $\mathrm{H}-2$ by an amino group. Consistently, the ${ }^{13} \mathrm{C}$ NMR spectrum revealed that there were 2 ketone groups ( $\delta 182.99$ and 181.90), suggesting that the naphthoquinone moiety is still present. Three additional aliphatic proton signals were found in the high field $(\delta 3.23$, 1.77, and 1.48-1.59), which belonged to $\mathrm{H}-2$ ', $\mathrm{H}-3^{\prime}$ and $\mathrm{H}-4$ '. The ${ }^{1} \mathrm{H}-{ }^{1} \mathrm{H}$ COSY spectrum confirmed the connections among them, and between $\mathrm{NH}-1^{\prime}$ and H-2' (Fig. 3). The position of the aliphatic chain was determined by the HMBC correlation of $\mathrm{NH}-1^{\prime}$ to $\mathrm{C}-1$, C-3 and C-2', and $\mathrm{H}-2$ ' to C-2 (Fig. 3). Thus, the structure of compound 11 was elucidated as 2,2'-(pentane-1,5-diylbis(azanediyl))bis(naphthalene-1,4-dione). The assigned carbon and proton signals are listed in Table 2.

Compound 12 was obtained as a purple powder, and its molecular formula was determined to be $\mathrm{C}_{13} \mathrm{H}_{9} \mathrm{NO}_{5} \mathrm{~S}$ by HRESIMS, which gave a $m / z 292.0276$ for $[\mathrm{M}+\mathrm{H}]^{+}$. The ${ }^{1} \mathrm{H}$ NMR spectrum showed 3 aromatic protons $(\delta 7.58,7.51$, and 7.25) and a chelating phenolic hydroxyl group $(\delta 12.61)$, while the ${ }^{13} \mathrm{C}$ NMR spectrum exhibited 2 ketone groups ( $\delta 184.20$ and 177.39) and an aromatic quaternary carbon signal belonged to C-5 at $\delta 160.61$ (Fig. S37 and S38). They established the 5-hydroxy-1,4-naphthoquinone core of 12 by a comparison of the NMR data with the substrate juglone (Fig. S4), [20] and it was further supported by the HMBC correlations shown in Fig. 3. The other additional proton signals were similar to those of $\mathbf{6}$ and $\mathbf{1 0}$, including a broad singlet at $\delta 8.08$ belonged to $\mathrm{NH}-1^{\prime}$, a $\mathrm{CH}$ multiplet at $\delta$ 4.44-4.61 belonged to $\mathrm{H}-2$ ', and 2 vicinal $\mathrm{CH}$ doublet of doublets both belonged to $\mathrm{H}-4$ ' ( $\delta 3.18$ and 3.05). The ${ }^{1} \mathrm{H}-{ }^{1} \mathrm{H}$ COSY correlations among them were shown in Fig. 3. Besides, similar to the ${ }^{1} \mathrm{H}$ NMR spectrum of 6 and 10, no signal for $\mathrm{H}-2$ or $\mathrm{H}-3$ was found. Those indicated that there was $\mathrm{Cys}$ moiety bridging $\mathrm{C}-2$ and $\mathrm{C}-3$ of the quinone ring. It was obvious that $\mathbf{1 2}$ was an additional product of $\mathrm{Cys}$ to juglone. The position of the Cys moiety was determined by the $\mathrm{HMBC}$ correlation of $\mathrm{NH}-1$ ' to $\mathrm{C}-1$, and H-4' to C-3 (Fig. 3). Thus, 12 was identified as 9hydroxy-5,10-dioxo-3,4,5,10-tetrahydro-2H-naphtho[2,3-b] [1, 4]thiazine-3-carboxylic acid. All the carbon and proton signals were assigned and listed in Table 2.

Biosynthesis of naphthoquinone derivatives supplemented with amino acids. Analyzing the structures of 1-15, most of the products seem to be the direct additions of amino acids to naphthoquinones. To confirm this, we added L-Glu into the fermentation broth of $S$. cerevisiae INVSc1 with supplemented 1,4-naphthoquinone to observe whether the yield of 9 increased. As a result, the content of 9 significantly increased, but we also detected it in substrate control (Fig. 4a). These suggested that these compounds were the additional products of amino acids with naphthoquinones. However, this fact alone was not enough to explain the formation of compound 6, 7, 10, 11, 12, 14 and 15. Among these compounds, 6, 10 and 12 had similar structures and $\mathbf{6}$ was the simplest one. The directed biosynthesis of $\mathbf{6}$ was carried out to explore the form of the dihydro-thiazine ring in their structure. Therefore, L-Cys was fed into the fermentation broth of $S$. cerevisiae INVSc1 with supplemented 1,4-naphthoquinone. After 7 days, the cultures were extracted and subjected to HPLC analysis. As shown in Fig. 4b, the production of 6 significantly increased as expected, compared to the fermentation broth without L-Cys (trace $\mathrm{i}$ and ii). However, 6 was also detected in substrate control (trace iii). This result explained the formation of dihydro-thiazine ring in 6, 10 and 12. Compounds 7, 14 and $\mathbf{1 5}$ may be the decarboxylation products after Ser molecule attached to 1,4-naphthoquinone or juglone. Considering $\mathbf{7}$ is the simplest compound among them and produced both by $S$. cerevisiae INVSc 1 and $E$. coli BL21(DE3), we next tested whether supplement of L-Ser in the fermentation broth with 1,4naphthoquinone can increase the production of 7 . Meanwhile, $\mathbf{1 1}$ is most likely biosynthesized by decarboxylation after Lys attached to two 1,4-naphthoquinones, so we also added L-Lys monohydrochloride into the fermentation broth of $E$. coli BL21(DE3) with supplemented 1,4-naphthoquinone to confirm this. The experiments were carried out for 7 days and the results were shown in Figs. 4c-4e. Unexpectedly, the productions of 7 and $\mathbf{1 1}$ not significantly increased as that of 6. Instead, 2 additional products, 16 and 17, were produced in significant amount. Besides, we also detected them in substrate control. Thus, $\mathbf{1 6}$ and $\mathbf{1 7}$ might be Ser and Lys attached to 1,4-naphthoquinone on the C-2 position, respectively. To confirm this, we purified them for structural characterization.

Structural characterization of 16 and 17. Compound 16 was isolated as an orange powder, exhibiting the molecular formula $\mathrm{C}_{13} \mathrm{H}_{11} \mathrm{NO}_{5}$ as established by HRESIMS $[\mathrm{M}+\mathrm{H}]^{+}, m / z$ 262.0711, which is one $\mathrm{CHO}_{2}$ unit longer than 7. Consistently, the ${ }^{13} \mathrm{C} \mathrm{NMR}$ spectrum of $\mathbf{1 6}$ revealed that there was an additional carboxyl carbon signal at $\delta 171.44$ (Fig. S47). These indicated that 16 may be Ser attached to 1,4-naphthoquinone on the $\mathrm{C}-2$ positions. The ${ }^{1} \mathrm{H}$ NMR spectrum of 16 showed several proton signals in the low field, including 4 aromatic proton signals $(\delta 8.03,7.95,7.85$, and 7.76), NH doublet $(\delta$ 6.98) and $\mathrm{CH}$ singlet $(\delta$ 5.74) (Fig. S46), which indicated the presence of a naphthoquinone moiety with $\mathrm{NH}$ at $\mathrm{C}-2$. CH multiplet at $\delta 4.18-4.26$ and $\mathrm{CH} 2$ doublet at $\delta 3.83$ that belong to $\mathrm{H}-2$ ' and $\mathrm{H}-4$ ' were observed in the high field. The ${ }^{1} \mathrm{H}-{ }^{1} \mathrm{H}$ COSY spectrum confirmed the connectivities among them and NH-1' (Fig. 3). Only one proton signal for H-2' was found, suggesting the substitution of a proton by a carboxyl group at this posi- 
(a)
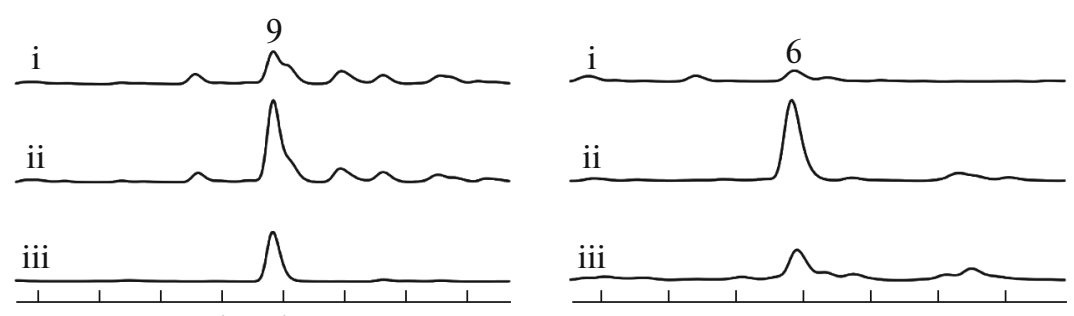

$14.515 .015 .516 .016 .517 .017 .518 .0 \min 18.018 .519 .019 .520 .020 .521 .0 \mathrm{~min}$

(d)

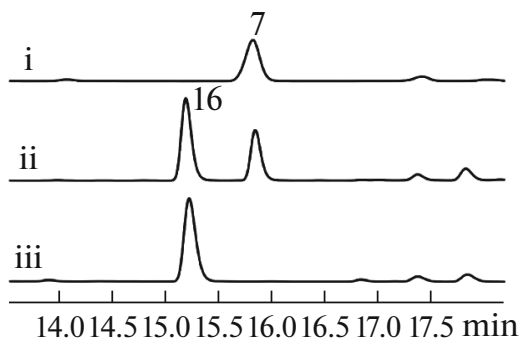

(b)

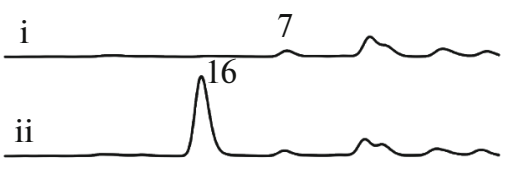

$14.014 .515 .015 .516 .016 .517 .0 \mathrm{~min}$ ii

(c)

(e)

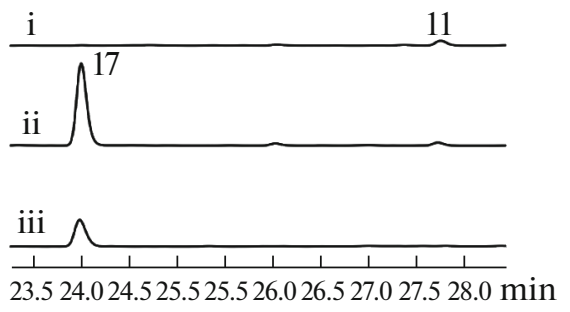

Fig. 4. HPLC (336 nm) analysis after incubation of 1,4-naphthoquinone and Glu, Cys or Ser with chassis cells. (a): i-S. cerevisiae INVSc1 + 1,4-naphthoquinone; ii-S. cerevisiae INVSc1 + 1,4-naphthoquinone + Glu; iii-1,4-naphthoquinone + Glu. (b): i-S. cerevisiae INVSc1 + 1,4-naphthoquinone; ii-S. cerevisiae INVSc1 + 1,4-naphthoquinone + Cys; iii-1,4-naphthoquinone + Cys. (c): i-S. cerevisiae INVSc1 + 1,4-naphthoquinone; ii-S. cerevisiae INVSc1 + 1,4-naphthoquinone + Ser; iii-1,4-naphthoquinone + Ser. (d): i-E. coli BL21(DE3) + 1,4-naphthoquinone; ii-E. coli BL21(DE3) + 1,4-naphthoquinone + Ser; iii-1,4-naphthoquinone + Ser. (e): i-E. coli BL21(DE3) + 1,4-naphthoquinone; ii-E. coli BL21(DE3) + 1,4-naphthoquinone + Lys; iii-1,4-naphthoquinone + Lys. Samples were analyzed using linear gradient of 10-90\% (vol/vol) acetonitrile-water (containing $0.1 \%$ formic acid) for $30 \mathrm{~min}$ at a flow rate of $1 \mathrm{~mL} / \mathrm{min}$.

tion, which was determined by the HMBC correlation of NH-1' to C-1, C-3 and C-3' (Fig. 3). Thus, the structure of $\mathbf{1 6}$ was elucidated as 2-(1,4-dioxo-1,4dihydronaphthalen-2-ylamino)-3-hydroxypropanoic acid. All the carbon and proton signals were assigned and are listed in Table 2.

Compound 17 was obtained as a red powder, determined the molecular formula as $\mathrm{C}_{26} \mathrm{H}_{22} \mathrm{~N}_{2} \mathrm{O}_{6}$ according to its HRESIMS data $\left([\mathrm{M}+\mathrm{H}]^{+}, m / z 459\right.$. 1561, calculated for $\left.\mathrm{C}_{26} \mathrm{H}_{23} \mathrm{~N}_{2} \mathrm{O}_{6}, 459.1556\right)$. 17 is one $\mathrm{CHO}_{2}$ unit longer than 11, suggesting that it may a derivative of $\mathbf{1 1}$ and contain a carboxyl group. Analysis of the ${ }^{1} \mathrm{H}$ NMR spectrum showed a carboxyl proton signal $(\delta 13.15), 8$ aromatic proton signals $(\delta 8.00-$ $7.88,7.82$, and 7.71), $2 \mathrm{NH}$ proton signals $(\delta 7.56$ and $7.25)$ and $2 \mathrm{CH}$ singlets $(\delta 5.69$ and 5.67) in the low field (Fig. S51), indicating the presence of a carboxyl group and 2 amino naphthoquinone moieties. In the high field of ${ }^{1} \mathrm{H}$ NMR spectrum, a methine multiplet $(\delta 4.11-4.19)$ and 4 methylene groups $(\delta 3.19,1.96$, 1.63 and 1.42) that belong to $\mathrm{H}-2^{\prime}, \mathrm{H}-7^{\prime}, \mathrm{H}-4^{\prime}, \mathrm{H}-6^{\prime}$ and $\mathrm{H}-5^{\prime}$ were observed. The ${ }^{1} \mathrm{H}-{ }^{1} \mathrm{H}$ COSY correlations among them and $2 \mathrm{NH}$ groups were shown in Fig. 3, suggesting that a long side chain is connected to 2 naphthoquinones via $2 \mathrm{NH}$ groups. Consistently, the ${ }^{13} \mathrm{C}$ NMR and HSQC spectra of $\mathbf{1 7}$ exhibited signals attributable to 2 naphthoquinone moieties, a carboxyl group $(\delta$ 173.04), a methine carbon $(\delta 55.13)$ and 4 methylene groups $(\delta 42.06,30.40,27.25$, and 23.31$)$ (Fig. S52 and S54). The carboxyl group was located at C-3' based on the HMBC correlations of NH-1', H-2' and $\mathrm{H}-4$ ' to $\mathrm{C}-3^{\prime}$. The linkages of the long side chain to 2 naphthoquinones were determined based on the HMBC correlations of $\mathrm{NH}-1^{\prime}$ at $\delta 7.25$ to $\mathrm{C}-1, \mathrm{C}-3$, C-2', C-3' and C-4', H-2' at $\delta 4.11-4.19$ to C-2, H-7' at $\delta 3.19$ to $\mathrm{C}-12$, and $\mathrm{NH}-8^{\prime}$ at $\delta 7.56$ to $\mathrm{C}-11, \mathrm{C}-13$, C-6' and C-7' (Fig. 3). Therefore, 17 was identified as 2,6-bis(1,4-dioxo-1,4-dihydronaphthalen-2-ylamino)hexanoic acid. The assigned carbon and proton signals are listed in Table 2.

Biosynthesis of amino sidechains for novel naphthoquinones derivatives. In order to confirm that $\mathbf{1 0}$ was biosynthesized from $\mathbf{6}$ and Gly, 7 was the decarboxylation product of $\mathbf{1 6}$, and $\mathbf{1 1}$ was the decarboxylation product of 17, we added the products as substrates into the fermentation broths of microorganisms. Since $\mathbf{1 0}$ was a derivative of $\mathbf{6}$ that contains an additional Gly residue connected to the Cys via an amide bond, $\mathbf{6}$ and Gly were fed into the fermentation broth of $S$. cerevisiae INVSc1 to produce 10. However, the peak for $\mathbf{1 0}$ was undetectable (Fig. 5a, iii). Compound 7 can be detected in both fermentation broths of $S$. cerevisiae INVSc1 and E. coli BL21(DE3), thus, we added 16 into these fermentation broths. The result was shown in Figs. 5b, 5c. As a result, 7 was produced, but the 
(a)

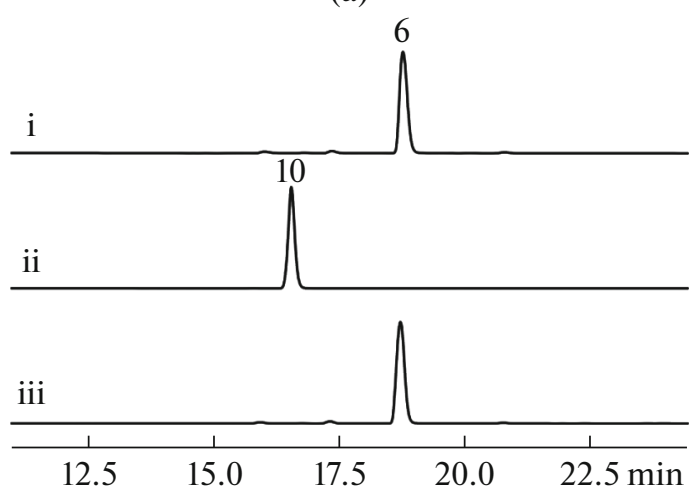

(c)

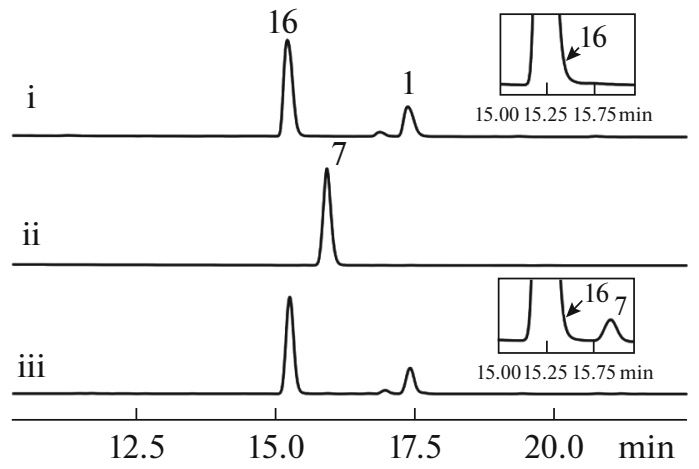

(b)

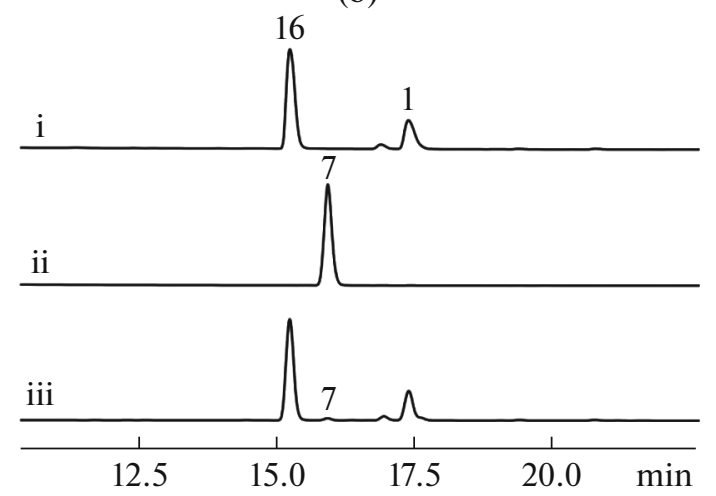

(d)

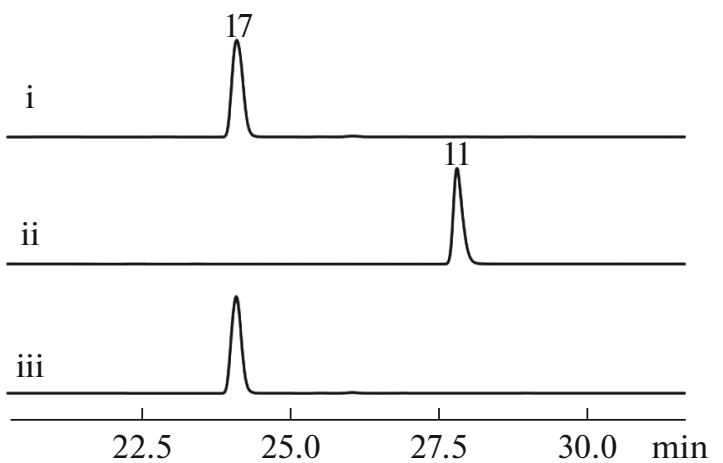

Fig. 5. HPLC analysis after incubation of 1,4-naphthoquinone derivatives and Gly with chassis cells. (a; $524 \mathrm{~nm}): \mathrm{i}-6+\mathrm{Gly}$ as the blank control; ii-10 in pure form; iii - S. cerevisiae INVSc1 + $\mathbf{6}+$ Gly. (b; $457 \mathrm{~nm}$ ): i-16 as the blank control; ii-7 in pure form; iii-S. cerevisiae INVSc1 + 16. (c; $457 \mathrm{~nm})$ : i-16 as the blank control; ii-7 in pure form; iii-E. coli BL21(DE3) + 16. (d; $456 \mathrm{~nm}$ ): i-17 as the blank control; ii-11 in pure form; iii-E. coli BL21(DE3) + 17. Samples were analyzed using linear gradient of $10-90 \%$ (vol/vol) acetonitrile-water (containing $0.1 \%$ formic acid) for $30 \mathrm{~min}$ at a flow rate of $1 \mathrm{~mL} / \mathrm{min}$.

production was low. This result indicated that 7 was indeed converted from 16, but the low titer suggested that 7 might be mainly produced through other pathways. In addition, we also observed 1 in the blank control of 16, suggesting that $\mathbf{1}$ may be the degradation product of these amino acid naphthoquinones. Compound 11 similarly seemed to be biosynthesized by the decarboxylation of 17, and next we tested the biotransformation of 17 by $E$. coli BL21(DE3). As shown in Fig. 5d, no peak for $\mathbf{1 1}$ was detected, indicating that $\mathbf{1 1}$ was not the decarboxylation product of $\mathbf{1 7}$.

Proposed biosynthetic pathway for the biosynthesis of naphthoquinone derivatives. In this work, we studied the modification of 1,4-naphthoquinone and juglone with the metabolites generated by $S$. cerevisiae or $E$. coli chassis cells. Seventeen products, including 6 novel compounds, were identified. As demonstrated in Fig. 7, we proposed the synthetic pathways for products $1-17$. Some compounds, including $\mathbf{2 - 5 , 8}$ and $\mathbf{9}$, were derived from naphthoquinones and contained an amino acid residue connected to $\mathrm{C}-2$ of the quinone ring. 2 was formed from Val, 3 from $\beta$-Ala, 4 from $\gamma$-aminobutyric acid, 5 from Ala, 8 from Gly and $\mathbf{9}$ from Glu. It was confirmed that compound 9 was generated by the incubation of Glu with 1,4-naphthoquinone. Compounds 6,10 and 12 share a similar structure, which have Cys moiety bridging C-2 and C-3 of the quinone ring through the amino and sulfhydryl groups to form a dihydro-thiazine ring. The incubation of $\mathbf{6}$ with Gly did not give 10, yet it was proposed that $\mathbf{1 0}$ was derived from the Cys-Gly dipeptide attached to 1,4-naphthoquinone on C-2 position because Cys-Gly dipeptide is generated from the glutathione S-transferase degradation pathway endogenous in S. cerevisiae [21].

Ethanolamine can be generated from the decarboxylation of Ser [22]. Compound 7 is derived from the attachment of ethanolamine to the $\mathrm{C}-2$ position of 1,4-naphthoquinone, whereas 14 and 15 were generated by the attachment of ethanolamine to the C-2 or C-3 position of juglone. The incubation of Ser with 1,4-naphthoquinone gave 16, which was further decarboxylated into 7 by both $S$. cerevisiae INVSc1 and $E$. coli BL21(DE3) chassis cells. 16 was nonenzymatically converted to 1 , suggesting that 13 was also the degradation product of juglone derivatives, such as $\mathbf{1 4}$ or 15. Compound $\mathbf{1 7}$ was generated by 2 units of 1,4-naphthoquinone attached to the amino groups of Lys. Incubation of 17 with E. coli BL21(DE3) did not 
Table 3. Binding energies of ligands docked against pocket 1 and 2 of Nsp9 of SARS-Cov-2

\begin{tabular}{c|c|c}
\hline Compounds & $\begin{array}{c}\text { Binding Affinity } \\
\text { for Pocket 1 }\end{array}$ & $\begin{array}{c}\text { Binding Affinity } \\
\text { for Pocket 2 }\end{array}$ \\
\hline 1 & -5.8 & -5.7 \\
3 & -6.2 & -6.2 \\
4 & -6.0 & -5.9 \\
5 & -5.9 & -5.9 \\
6 & -6.4 & -6.1 \\
7 & -5.8 & -6.5 \\
8 & -5.8 & -5.7 \\
9 & -6.1 & -6.0 \\
10 & -6.2 & -6.2 \\
$\mathbf{1 1}$ & -6.1 & -6.7 \\
12 & -7.5 & -6.7 \\
13 & -5.9 & -6.4 \\
14 & -5.4 & -5.6 \\
15 & -5.3 & -5.6 \\
16 & -5.5 & -5.7 \\
$\mathbf{1 7}$ & -6.2 & -6.0 \\
\hline & -5.9 & -7.9 \\
\hline
\end{tabular}

give 11, indicating that $\mathbf{1 1}$ was not the decarboxylation product of 17. Free Lys in E. coli may have been directly converted to cadaverine by lysine decarboxylase before synthesizing 11 [23]. Thus, two units of 1,4-naphthoquinone were connected by cadaverine on the amino groups.

Biological activities of naphthoquinones and the investigation of potential antiviral activities of 1-17 using molecular docking. 1,4-naphthoquinone pharmacophore is known to impart pronounced biological effects on its derivatives, leading to antitumor, antimalarial, antiviral, molluscidal, antileishmanial, antiproliferative, antibacterial and antifungal activities [2]. Among 17 compounds identified, 9-12, 16 and 17 are novel compounds reported firstly in this study, which have potential to be bioactive agents. Most of the known compounds obtained in this work have been proven to be biologically active compounds. For instance, $\mathbf{1}$ has various antimicrobial, anticancer and antimalarial activities. As an antimicrobial agent, it showed strong activity against Gram-negative bacteria (Salmonella typhimurium, Acinetobacter baumanii, Citrobacter freundii and Proteus mirabilis), Gram-positive bacteria (Staphylococcus aureus), and the yeast (Candida albicans) [24]. The anticancer activity of naphthoquinone derivatives was tested on pancreatic cancer MIA PaCa-2 cells and some derivatives were reported to be promising anticancer agents [25]. 2 was also demonstrated antimicrobial activity, but it was less active than 1,4-naphthoquinone [26]. Anticancer activities of 3-5 and 8 were previously reported.
Importantly, $\mathbf{5}$ and $\mathbf{8}$ showed high cytotoxic activity and selectivity against 8 human cancer cell lines [5]. In addition, 8 was also reported to have antimycobacterial activities, but the specificity was low [27]. 7 possessed cytotoxic activity against lung cancer cell line H460. [28] 14 exhibited significant cytotoxicity towards cultured MDA-MB231, HepG2 and SNU638 cells [29].

The antiviral activities of naphthoquinones were also reported. For example, 2 novel naphthoquinones, isolated from a medicinal plant Rhinacanthus nasutus (L.) Kurz (Acanthaceae), exhibited inhibitory activity against cytomegalovirus, with EC50 values of 0.02 and $0.22 \mu \mathrm{g} / \mathrm{mL}$ [30]. Another new naphthoquinone extracted from the same medicinal plant was tested against coxsackievirus B3, human rhinovirus $1 \mathrm{~B}$ and influenza PR8 virus and exhibited strong antiviral activity with the IC50 value ranging from 0.03 to $23.7 \mu \mathrm{M}$ [31]. Taldon et al. also investigated the antiviral activities of novel 1,4-naphthoquinone derivatives, 2 of which showed strong antiviral activity against influenza-A and herpes simplex viruses [32].

More interestingly, lapachol and its derivatives, which are naphthoquinone derivatives, were recently tested against SARS-CoV-2 using in silico approaches [6]. In this study, GOLD program was used for molecular docking against Nsp9 of SARS-CoV-2 and lapachol derivative IX showed the highest docking score. Therefore, it was expected for the compounds biosynthesized in this study to possess similar bioactivities. To justify this, in silico approach was simply conducted to screen the potential antiviral activities of compounds 1-17 against Nsp9 of SARS-CoV-2. These compounds were subjected to molecular docking. The druggability and pocket prediction were performed using an online protein binding cavity analyzer (http:// www.pkumdl.cn:8000/cavityplus/computation.php). The most druggable 2 pockets were targeted for molecular docking. The pocket 1 covered the Nsp9specific $\alpha$-helical GxxxG dimerization interface [16]. The pocket 2 covered the amino acid residues that are actively involved in the RNA binding site of Nsp9. Both pockets are important targets for the inhibition of Nsp9. GxxxG motif is considered important for viral replication and its disruption of Nsp9 of porcine delta coronavirus blocked nucleotide binding capacity [33]. RNA binding site of Nsp9 was reported as a promising drug target to discover potential antiviral compounds. With this approach, potential compounds were identified against dengue virus, Chikungunya virus and SARS-CoV [6]. After specifying the grid boxes based on predicted druggable pockets, protein and ligand preparations were completed and 6W4B were docked against 1-17 using Autodock Vina. Binding energies $(\mathrm{kcal} / \mathrm{mol})$ of ligands docked against both pockets are listed in Table 3. Compound $\mathbf{1 1}$ had the highest binding affinity for the pocket 1 of Nsp9, while 17 showed the highest binding affinity for pocket 2 . The interaction of these two compounds with their corresponding 


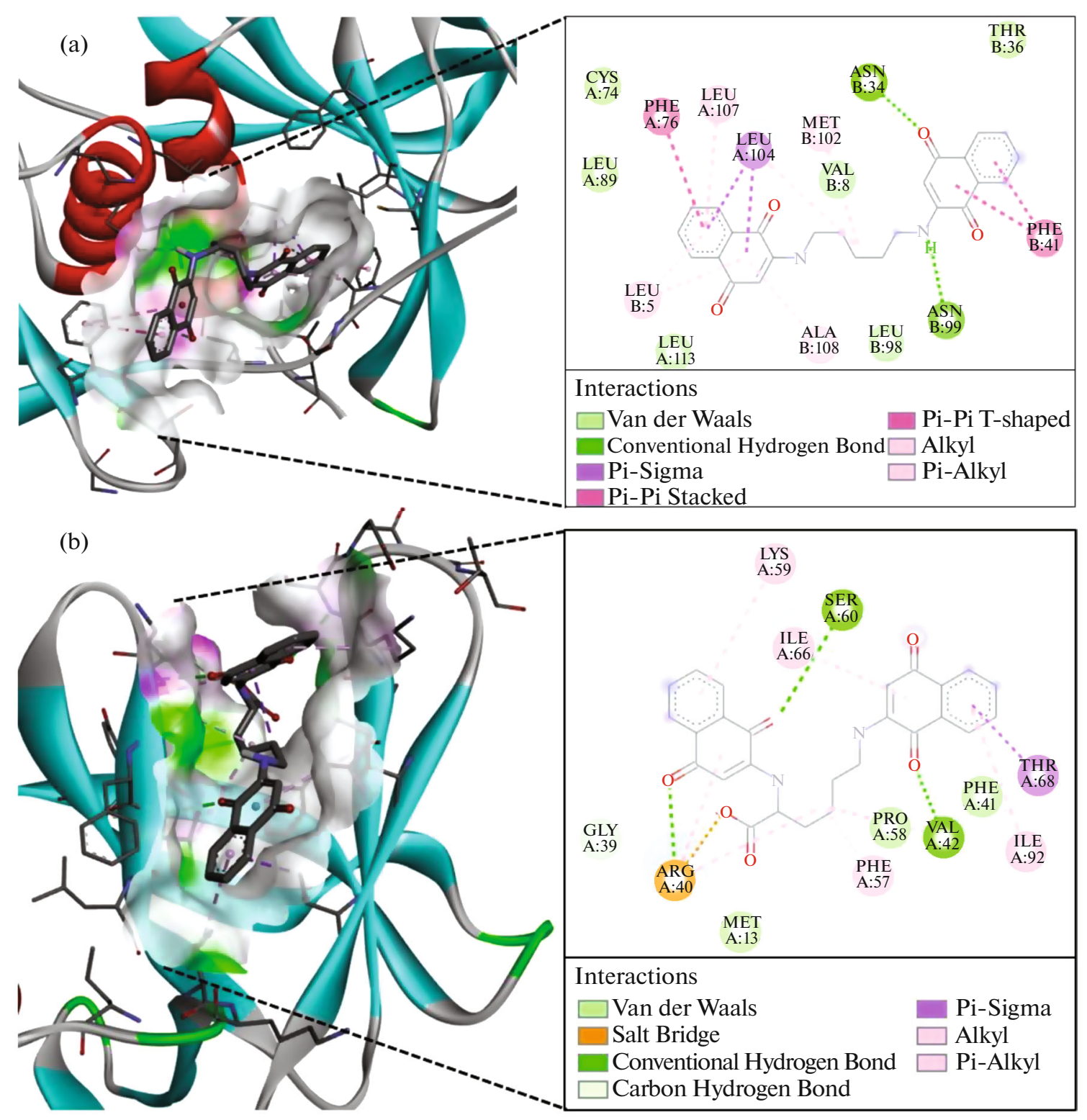

Fig. 6. 3D visualization of protein-ligand complexes and 2D diagrams of interactions for selected ligands and pockets with their best affinity poses. (a) Compound 11 docked against pocket 1 of Nsp9. (b) Compound 17 docked against pocket 2 of Nsp9.

pockets of Nsp9 was illustrated in Fig. 6. 11 formed 2 hydrogen bonds to Asn34-B and Asn99-B (-A: on chain $\mathrm{A}$ and -B: on chain $\mathrm{B}$ ) as well as several hydrophobic (Leu5-B, Val8-B, Phe41-B, Phe76-A, Met102-B, Leu104-A, Leu107-A and Ala108-A) and van der Waals interactions (Val8-B, Thr36-B, Cys74A, Leu89-A, Leu98-B and Leu113-A) (Fig. 6a). On the other hand, 17 had 3 hydrogen bonds to Arg40-A, Val42-A and Ser60-A and formed a salt bridge to Arg40-A. Several hydrophobic (Arg40-A, Val42-A, Phe57-A, Lys59-A, Ile66-A, Thr68-A and Ile92-A) and van der Waals interactions (Met13-A, Gly39-A Phe48-A and Pro58-A) were also observed for 17. Additionally, Gly39-A formed a carbon-hydrogen bond with 17 at the quinone subunit of symmetrical 1,4-naphtoquinone (Fig. 6b). To compare our results with the literature, the molecular docking experiments for the lapachol IX derivative was performed and resulted in binding affinities of -6.5 and -7.7 to pocket 1 and 2, respectively. The best docking scores of our compounds for each pocket outperformed this compound. Thus, it is expected that $\mathbf{1 1}$ and $\mathbf{1 7}$ hold promise to be the antiviral compounds for the treatment of SARS-CoV-2. However, this preliminary finding needs to be confirmed by molecular dynamics simulations, in vitro and in vivo experiments to draw a solid and more reliable conclusion.

Our work demonstrated that the metabolites of $S$. cerevisiae and $E$. coli chassis cells can modify the polyketides such as naphthoquinones. $\mathrm{Hu}$ et al. iso- 


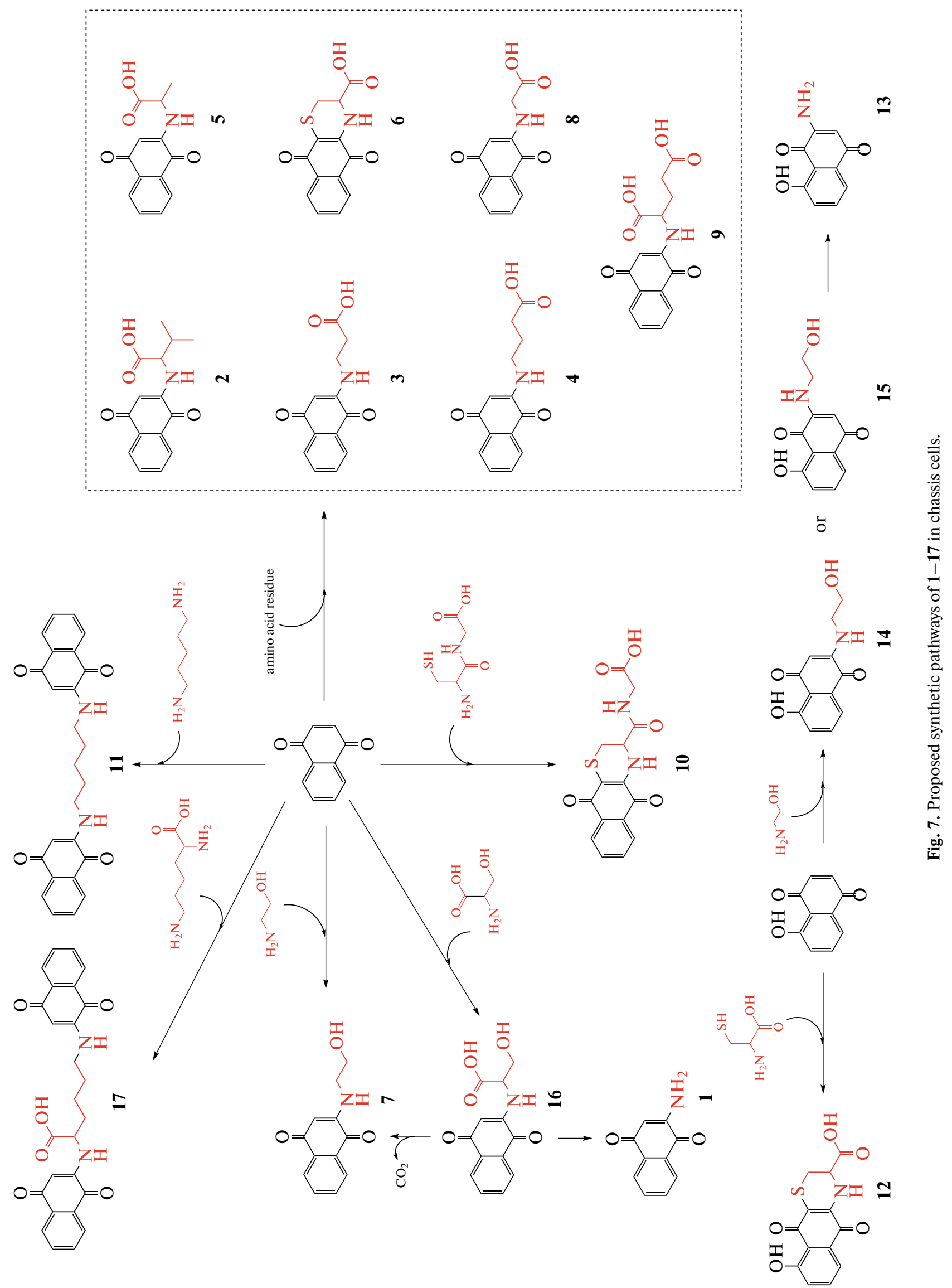


lated 2 shunt products in the synthesis of the perylenequinone elsinochrome A by Aspergillus nidulans, they were the 1,2-naphthoquinones adduct of Cys and Cys-Gly dipeptide [34]. Lebeau et al. detected 9 nitrogen-containing azaphilone red pigments from a wild type of Talaromyces sp. 30570 marine-derived fungal strain, 6 among which were occurred only when complex organic nitrogen sources were present in the culture medium. These structures contained an amino acid residues [35]. Sun et al. also demonstrated the influences of exogenous glucose and pyruvate on the polyketide product flaviolin in an engineered $E$. coli BL21(DE3) [19]. Therefore, as heterologous expression host, some certain intracellular metabolites of $S$. cerevisiae and E. coli chassis cells may modify the target products to create chemical diversity. This not only provides a new approach for the biosynthesis of novel molecules in the commonly used chassis cells, but also expands the chemical repertoire for the discovery of novel and promising agents with various biological activities such as anticancer and anti-SARS-CoV-2.

\section{FUNDING}

This research was supported by National Natural Science Foundation of China (Grant no. 31800667), Project of south medicine innovation team in modern agricultural industry technology system of Guangdong Province (Grant no. 2020KJ148), Guangdong Province Innovative and Strong School Project Scientific Research Project (Grant no. 2017KTSCX045).

\section{COMPLIANCE WITH ETHICAL STANDARDS}

The authors declare that they have no conflicts of interest. This article does not contain any studies involving animals or human participants performed by any of the authors.

\section{AUTHOR CONTRIBUTIONS}

$\mathrm{W}$. Wu and S. Wang contributed to this work equally.

\section{SUPPORTING INFORMATION}

Supporting information may be found in the online version of this article.

\section{SUPPLEMENTARY INFORMATION}

The online version contains supplementary material available at https://doi.org/10.1134/S0003683821100124.

\section{REFERENCES}

1. Qiu, H.Y., Wang, P.F., Lin, H.Y., Tang, C.Y., Zhu, H.L., and Yang, Y.H., Chem. Biol. Drug Des., 2018, vol. 91, no. 3, pp. 681-690.
2. Sharma, U., Katoch, D., Sood, S., Kumar, N., Singh, B., Thakur, A., and Gulati, A., Indian J. Chem. Sect. B, 2013, vol. 52, no. B, pp. 1431-1440.

3. Pereyra, C.E., Dantas, R.F., Ferreira, S.B., Gomes, L.P., and Silva, F.P., Jr., Cancer Cell Int., 2019, vol. 19, no. 1, pp. 1-20.

4. Xavier, M.R., Santos, M.M.S., Queiroz, M.G., De Lima Silva, M.S., Goes, A.J.S., and De Morais, M.A., Jr., Mol. Biol. Rep., 2020, vol. 47, no. 2, pp. 1173-1185.

5. De Moraes, T.A., Filha, M.J., Camara, C.A., Silva, T., Soares, B.M., Bomfim, I.S., et al., Molecules, 2014, vol. 19, no. 9, pp. 13188-13199.

6. Junior, N.N., Santos, I.A., Meireles, B.A., Nicolau, M.S.A.P., Lapa, I.R., et al., J. Biomol. Struct. Dyn., 2021, vol. 2021, pp. 1-15.

7. Bin, L., Xu, G., Wang, Z.-Q., Ye, D., Chen, W., Luo, T.-F., et al., Pharmacol. Sin., 2017, vol. 38, no. 11, pp. 1543-1553.

8. Zhang, Y., Xu, T., Pan, Z., Ge, X., Sun, C., Lu, C., et al., Br. J. Pharmacol., 2018, vol. 175, no. 5, pp. 840854.

9. Arasoglu, T., Mansuroglu, B., Derman, S., Gumus, B., Kocyigit, B., Acar, T., and Kocacaliskan, I., J. Agric. Food Chem., 2016, vol. 64, no. 38, pp. 7087-7094.

10. Reddy, P.J., Ray, S., Sathe, G.J., Prasad, T.K., Rapole, S., Panda, D., and Srivastava, S., Omics, 2015, vol. 19, no. 1, pp. 12-23.

11. Walther, B., Karl, J.P., Booth, S.L., and Boyaval, P., Adv. Nutr., 2013, vol. 4, no. 4, pp. 463-473.

12. Ren, L., Peng, C., Hu, X., Han, Y., and Huang, H., Biotechnol. Adv., 2020, vol. 39, p. 107453.

13. Guzzetta, A.W., Clark, P., Tappe, R., and Budzikiewicz, H., Naturforsch. C, 1999, vol. 54, nos. 3-4, pp. $175-180$.

14. Fidan, O. and Zhan, J., Molecular Pharming: Applications, Challenges, and Emerging Areas, Kermode, A.R. and Liang, L., Eds., New Jersey: Wiley Blackwell, 2018.

15. Moses, T., Mehrshahi, P., Smith, A.G., and Goossens, A., J. Exp. Bot., 2017, vol. 68, no. 15, pp. 4057-4074.

16. Littler, D.R., Gully, B.S., Colson, R.N., and Rossjohn, J., Science, 2020, vol. 23, no. 7, pp. 101258.

17. Morris, G.M., Huey, R., Lindstrom, W., Sanner, M.F., Belew, R.K., Goodsell, D.S., and Olson, A.J., J. Comput. Chem., 2009, vol. 30, no. 16, pp. 2785-2791.

18. Trott, O. and Olson, A.J., J. Comput. Chem., 2010, vol. 31, no. 2, pp. 455-461.

19. Sun, L., Zeng, J., Zhang, S., Gladwin, T., and Zhan, J., Appl. Microbiol. Biotechnol., 2015, vol. 99, no. 2, pp. 693-701.

20. Suchard, O., Kane, R., Roe, B.J., Zimmermann, E., Jung, C., Waske, P.A., et al., M., Tetrahedron, 2006, vol. 62, no. 7, pp. 1467-1473.

21. Kaur, H., Ganguli, D., and Bachhawat, A.K., J. Biol. Chem., 2012, vol. 287, no. 12, pp. 8920-8931.

22. Yunus, I.S., Liu, Y.C., and Nakamura, Y., Plant J., 2016, vol. 88, no. 4, pp. 559-569. 
23. Goldemberg, S.H., J. Bacteriol., 1980, vol. 141, no. 3, pp. 1428-1431.

24. Rahmoun, N.M., Boucherit-Atmani, Z., Benabdallah, M., Boucherit, K., Villemin, D., and ChoukchouBraham, N., Am. J. Med. Biol. Res., 2013, vol. 1, no. 1, pp. 16-22.

25. Rozanov, D., Cheltsov, A., Nilsen, A., Boniface, C., Forquer, I., Korkola, J., et al., PLoS One, 2019, vol. 14, no. 3 , art. e0205623.

26. Janeczko, M., Demchuk, O.M., Strzelecka, D., Kubiński, K., and Masłyk, M., Eur. J. Med. Chem., 2016, vol. 124, pp. 1019-1025.

27. Mital, A., Sonawane, M., Bindal, S., Mahlavat, S., and Negi, V., Der. Pharma. Chem., 2010, vol. 2, no. 3, pp. 63-73.

28. Delarmelina, M., Daltoé, R.D., Cerri, M.F., Madeira, K.P., Rangel, L., Lacerda Júnior, V., et al., J. Braz. Chem. Soc., 2015, vol. 26, no. 9, pp. 1804-1816.
29. Jin, M., Sun, J., Li, R., Diao, S., Zhang, C., Cui, J., et al., Arch. Pharm. Res., 2016, vol. 39, no. 9, pp. 12371241.

30. Sendl, A., Chen, J.L., Jolad, S., Stoddart, C., Rozhon, E., Kernan, M., et al., J. Nat. Prod., 1996, vol. 59, no. 8, pp. 808-811.

31. Ngoc, T.M., Phuong, N.T.T., Khoi, N.M., Park, S., Kwak, H.J., Nhiem, N.X., et al., Nat. Prod. Res., 2019, vol. 33, no. 3, pp. 360-366.

32. Tandon, V.K., Singh, R.V., and Yadav, D.B., Bioorg. Med. Chem. Lett., 2004, vol. 14, no. 11, pp. 2901-2904.

33. Miknis, Z.J., Donaldson, E.F., Umland, T.C., Rimmer, R.A., Baric, R.S., and Schultz, L.W., J. Virol. 2009, vol. 83, no. 7, pp. 3007-3018.

34. Hu, J., Sarrami, F., Li, H., Zhang, G., Stubbs, K.A., Lacey, E., et al., Chem. Sci., 2019, vol. 10, no. 5, pp. 1457-1465.

35. Lebeau, J., Petit, T., Fouillaud, M., Dufossé, L., and Caro, Y., Microorganisms, 2020, vol. 8, no. 12, p. 1920. 